\title{
Direct Parallel Computations of Second-Order Search Directions for Model Predictive Control
}

Isak Nielsen and Daniel Axehill

The self-archived postprint version of this journal article is available at Linköping University Institutional Repository (DiVA):

http://urn.kb.se/resolve?urn=urn:nbn:se:liu:diva-158940

N.B.: When citing this work, cite the original publication.

Nielsen, I., Axehill, D., (2019), Direct Parallel Computations of Second-Order Search Directions for Model Predictive Control, IEEE Transactions on Automatic Control, 64(7), 2845-2860.

https://doi.org/10.1109/TAC.2018.2880405

Original publication available at:

https://doi.org/10.1109/TAC.2018.2880405

Copyright: Institute of Electrical and Electronics Engineers (IEEE)

http://www.ieee.org/index.html

(C)2019 IEEE. Personal use of this material is permitted. However, permission to reprint/republish this material for advertising or promotional purposes or for creating new collective works for resale or redistribution to servers or lists, or to reuse any copyrighted component of this work in other works must be obtained from the IEEE. 


\title{
Direct Parallel Computations of Second-Order Search Directions for Model Predictive Control
}

\author{
Isak Nielsen and Daniel Axehill
}

\begin{abstract}
Model Predictive Control (MPC) is one of the most widely spread advanced control schemes in industry today. In MPC, a constrained finite-time optimal control (CFTOC) problem is solved at each iteration in the control loop. The CFTOC problem can be solved using for example second-order methods such as interior-point (IP) or active-set (AS) methods, where the computationally most demanding part often consists of computing the sequence of second-order search directions. These can be computed by solving a set of linear equations which corresponds to solving a sequence of unconstrained finite-time optimal control (UFTOC) problems. In this paper, different direct (non-iterative) parallel algorithms for solving UFTOC problems are presented. The parallel algorithms are all based on a recursive reduction and solution propagation of the UFTOC problem. Numerical evaluations of the proposed parallel algorithms indicate that a significant boost in performance can be obtained, which can facilitate high performance second-order MPC solvers.
\end{abstract}

Index Terms-MPC, optimization, parallel, Riccati recursion

\section{INTRODUCTION}

$\mathbf{I}$ N Model Predictive Control (MPC), the control input is computed by solving a constrained finite-time optimal control (CFTOC) problem at each iteration in the control loop. When a second-order method such as an interior-point (IP) or an active-set (AS) method is used to solve the CFTOC problem, the main computational effort is often spent on solving a sequence of second-order search directions. In the case of MPC, this corresponds to solving a sequence of unconstrained finite-time optimal control (UFTOC) problems. Hence, much effort in research has been spent on solving UFTOC problems efficiently using for example sparsity-exploiting algorithms or tailor-made algorithms such as the Riccati recursion. Some examples of such approaches are presented in [1], [2], [3], [4], [5], [6], [7], [8], [9].

One approach to speed up the computations of the search directions is to exploit parallelism. Two conceptually different ways to do so when solving CFTOC problems are to apply a parallel algorithm directly to the CFTOC problem, or to parallelize the computations of the search directions in the solver used to solve the CFTOC problem. Examples where the parallelism is applied directly to the CFTOC problem are [10], [11], [12], and [13]. In this paper, parallel algorithms for solving the UFTOC problems which correspond to computing the secondorder search directions are presented. In [14] the authors argue that exploiting parallelism like this at the level of numerical linear algebra is more flexible than exploiting it directly for the CFTOC problem. In [15] an extended Parallel Cyclic Reduction

I. Nielsen and D. Axehill are with the Division of Automatic Control, Linköping University, SE-58183 Linköping, Sweden, isak.nielsen@liu.se, danieldisy.liu.se. algorithm is used to reduce the computation of the UFTOC problem to smaller systems of equations that are solved in parallel. The computational complexity of this algorithm is reported to be $\mathcal{O}(\log N)$. In [16] and [17] a time-splitting approach where subproblems are connected through common variables and are solved in parallel using Schur complements is used. The common variables are computed via a consensus step where a dense system of equations involving all common variables is solved serially. In [18] and [19] a message-passing algorithm for IP methods is presented, which extends the approach introduced earlier in [20] to more general problems.

In this paper, structure-exploiting parallel algorithms for solving UFTOC problems are proposed. The algorithms can compute the solution in $\mathcal{O}(\log N)$ computational complexity and they exploit the special structure inherited from the UFTOC problem. Furthermore, it is shown how the Riccati recursion can be computed in parallel. The parallel algorithms presented in this paper can be put into a framework where a UFTOC problem of prediction horizon $N$ is reduced in parallel to a new, smaller master UFTOC problem in the same form but with prediction horizon $\hat{N}<N$. Unlike for example the partitioned dynamic programming algorithm in [14], the UFTOC problem structure is preserved in the master problem. Hence, the same parallel algorithm can be applied recursively, which facilitates an efficient implementation. Furthermore, in the algorithms presented here the solution is computed directly without involving any iterative updates of variables as is usually required in parallel first-order methods such as the alternating direction method of multipliers [21]. A more extensive and detailed presentation of the work presented here is available in the publication [22].

In this article, $\mathbb{S}_{++}^{n}\left(\mathbb{S}_{+}^{n}\right)$ denotes symmetric positive (semi) definite matrices with $n$ columns, $\mathbb{Z}_{i, j} \triangleq\{z \in \mathbb{Z} \mid i \leq z \leq j\}$ an interval of integers, $\mathcal{R}(A)$ the range space of a matrix $A$, and colrank $A$ the column rank of a matrix $A$.

The paper is organized as follows; in Section II the problem is formulated and in Section III the main steps of the parallel algorithms are outlined. In Section IV and Section V, the different ways of reducing and solving the UFTOC problem are presented. The recursive reduction framework is presented in Section VI and numerical results are presented in Section VII.

\section{Problem Formulation}

When for example IP and AS methods are applied to solve CFTOC problems, the computation of the search direction often corresponds to solving a UFTOC problem. In the general case, time-dependent matrices, linear and constant terms in the objective function, and an affine term in the dynamics equation 
are present. However, these are omitted here for presentational brevity and the reader is instead referred to [22] for the details when they are present. The UFTOC problem is then given by

$$
\begin{aligned}
\min _{\mathrm{x}, \mathrm{u}} & \sum_{t=0}^{N-1} \frac{1}{2}\left[\begin{array}{l}
x_{t} \\
u_{t}
\end{array}\right]^{T}\left[\begin{array}{cc}
Q_{x} & Q_{x u} \\
Q_{x u}^{T} & Q_{u}
\end{array}\right]\left[\begin{array}{l}
x_{t} \\
u_{t}
\end{array}\right]+\frac{1}{2} x_{N}^{T} Q_{x, N} x_{N} \\
\text { s.t. } & x_{0}=\bar{x} \\
& x_{t+1}=A x_{t}+B u_{t}, t \in \mathbb{Z}_{0, N-1} .
\end{aligned}
$$

Assumption 1: The Hessian of the objective function of the UFTOC problem (1) is positive semidefinite for all $t \in \mathbb{Z}_{0, N}$.

Assumption 2: $Q_{u}$ in (1) satisfies $Q_{u} \in \mathbb{S}_{++}^{n_{u}}$.

Unless stated otherwise, let Assumptions 1 and 2 hold, let $\mathcal{P}(N)$ denote an optimization problem with the same structure as in (1), and let the dual variables corresponding to the equality constraints in (1) be denoted $\lambda_{t}$ for $t \in \mathbb{Z}_{0, N}$.

One way of solving the UFTOC problem (1) is to use the Riccati recursion, see for instance [1], [2], [5], [22]. It consists of a factorization of the KKT matrix (see, e.g., [23]) of the UFTOC problem (Algorithm 1) followed by a state recursion to compute the solution (Algorithm 2). The computational complexity growth of the Riccati recursion is $\mathcal{O}\left(N\left(n_{x}+n_{u}\right)^{3}\right)$.
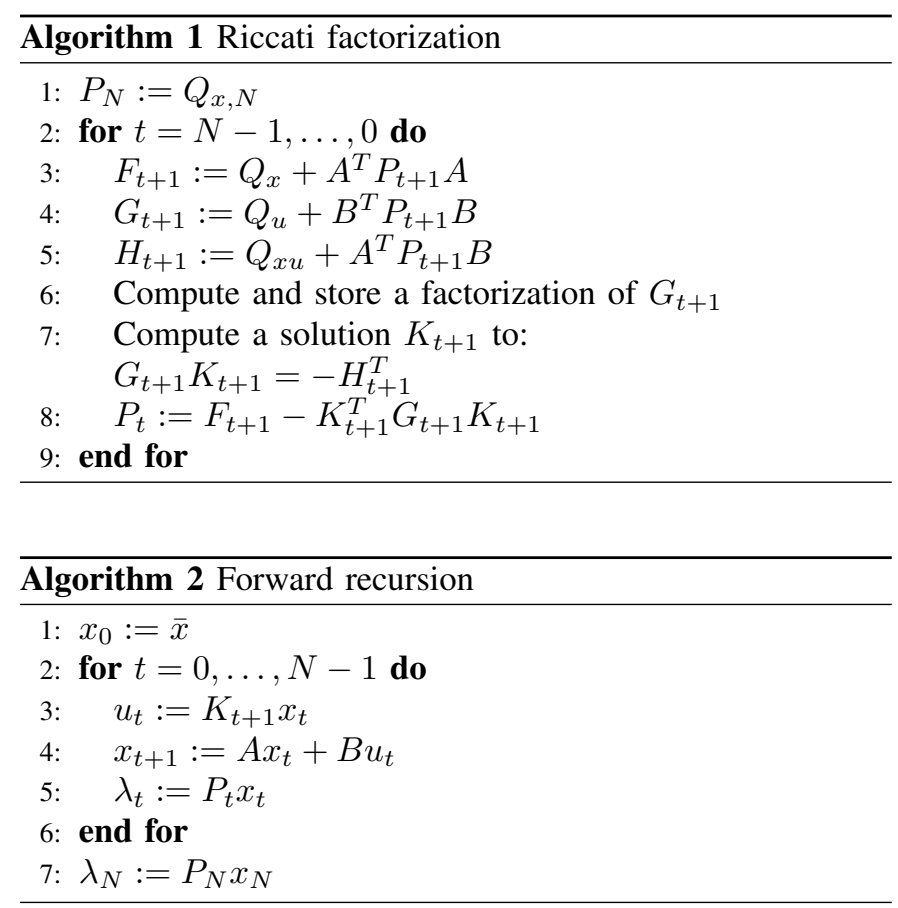

\section{Defining the Master Problem}

The original UFTOC problem (1) can be reduced to a master problem in the same UFTOC form but with shorter prediction horizon and possibly fewer control inputs using different techniques. Furthermore, the solution to the original UFTOC problem can be computed from the solution to the master problem in different ways. The reduction and solution of the original UFTOC problem can be described by four main steps:

1) Split into subproblems: Split the original UfTOC problem in time in $\hat{N}+1$ subproblems $\mathcal{P}_{i}\left(N_{i}\right)$ for $i \in \mathbb{Z}_{0, \hat{N}}$.

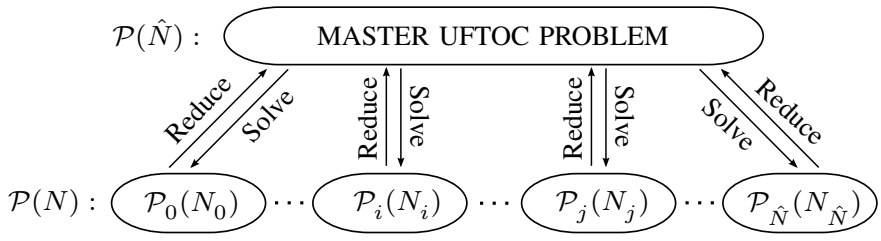

Fig. 1: The original UFTOC problem can be split into $\hat{N}+1$ smaller subproblems $\mathcal{P}_{i}\left(N_{i}\right)$ and reduced into a smaller master UFTOC problem. Once the master UFTOC problem is solved, the subproblems can be solved using information from the solution to the master UFTOC problem. The solution to the original UFTOC problem can be retrieved from the solutions to the subproblems $\mathcal{P}_{i}\left(N_{i}\right)$. The reduction and solution of the subproblems can be performed in different ways.

2) Reduce the subproblems: Reduce the subproblems to depend on fewer variables using any of the algorithms that will be presented in Section IV or Section V.

3) Form and solve the master problem: Form the master UFTOC problem $\mathcal{P}(\hat{N})$ using the reduced subproblems. Solve the master UFTOC problem.

4) Solve subproblems and retrieve solution: Solve the subproblems using information from the solution to the master UFTOC problem. Retrieve the solution to the original UFTOC problem $\mathcal{P}(N)$ using the solutions to the subproblems $\mathcal{P}_{i}\left(N_{i}\right)$ for $i \in \mathbb{Z}_{0, \hat{N}}$.

The structure of the reduction and solution process is presented in Fig. 1, where the dots represent repetition of the structure. The problem at the lower level is the split original UFTOC problem (1) and at the upper level the master UFTOC problem.

In Section IV, it will be shown how the reduction can be done using the parametric programming approach introduced in [20]. In Section V it is shown how the subproblems can be reduced by combining ideas from partial condensing, which was introduced in [24], with the theory introduced in [25]. The different approaches presented in this paper are similar in the sense that they split the original subproblem into several smaller subproblems, which can be reduced in parallel to eliminate variables from the original UFTOC problem. However, they differ in the way the problem is split, how the subproblems are reduced, and how the subproblems are solved. Depending on which reduction approach that is used, the solutions to the subproblems and the original UFTOC problem can be computed differently. The choice of approach may vary depending on the use of the solution.

The smaller subproblems are obtained by splitting the prediction horizon in $\hat{N}+1$ intervals $i \in \mathbb{Z}_{0, \hat{N}}$, where each is of length $N_{i} \in \mathbb{Z}_{++}$such that $\sum_{i=0}^{\hat{N}}=N$. Furthermore, let $t_{0}=0$ and $t_{i}=\sum_{k=0}^{i-1} N_{k}$ for $i \in \mathbb{Z}_{1, \hat{N}}$.

\section{Parametric Programming Approach}

The parallel approach introduced in [20] extends the original UFTOC problem with extra complicating variables, and splits it into smaller subproblems. In each subproblem (except the last), the final state has to satisfy a terminal constraint which involves the complicating variables. The connections between the subproblems are given by extra coupling constraints in 
the complicating variables, and the subproblems are solved parametrically as a function of these complicating variables.

This approach is similar to primal decomposition [26], [13], and to the hierarchical approach presented in [27] and [12] in the sense that the problem is decomposed in time into $\hat{N}+1$ subproblems that share complicating variables. Here, however, the parallel algorithm is applied at the level of the numerical linear algebra instead of to the inequality constrained problem. Furthermore, the introduction of the complicating variables and constraints is made such that feasible subproblems are always obtained, and the master problem is in the form of a UFTOC problem. Hence, the proposed parallel algorithm can be applied recursively without making any modifications to it.

\section{A. Problem decomposition and variable elimination}

The structure of the UFTOC problem (1) can be exploited by splitting it into subproblems that only share a small number of complicating variables. To do so, introduce the local variables

$$
\mathrm{x}_{\mathrm{i}} \triangleq\left[\begin{array}{lll}
x_{0, i}^{T} & \ldots & x_{N_{i}, i}^{T}
\end{array}\right]^{T}, \quad \mathrm{u}_{\mathrm{i}} \triangleq\left[\begin{array}{lll}
u_{0, i}^{T} & \ldots & u_{N_{i-1}, i}^{T}
\end{array}\right]^{T},
$$

the initial constraints $x_{0, i}=\hat{x}_{i}$ for each subproblem, and the terminal constraints $x_{N_{i}, i}=d_{i}$ for $i \in \mathbb{Z}_{0, \hat{N}-1}$. Here, $d_{i}$ will be used as a dummy variable in an intermediate step. The connections between the subproblems are introduced as the coupling constraints $\hat{x}_{i+1}=d_{i}$ for $i \in \mathbb{Z}_{0, \hat{N}-1}$. Now, let $d_{i}$ be parametrized as

$$
d_{i}=\hat{A} \hat{x}_{i}+\hat{B} \hat{u}_{i}
$$

where $\hat{x}_{i} \in \mathbb{R}^{n_{x}}$ and $\hat{u}_{i} \in \mathbb{R}^{n_{\hat{u}}}$ with $n_{\hat{u}} \leq n_{x}$ are introduced as the complicating variables. How, and also the reason why, to choose the parametrization $\hat{A}$ and $\hat{B}$ will soon be explained.

Now, define the number of variables $(n)$, equality constraints $(m)$ and parameters $(p)$ for $i \in \mathbb{Z}_{0, \hat{N}-1}$ as

$n \triangleq\left(N_{i}+1\right) n_{x}+N_{i} n_{u}, \quad m \triangleq\left(N_{i}+2\right) n_{x}, \quad p \triangleq n_{x}+n_{\hat{u}}$.

Furthermore, define $\mathrm{H} \in \mathbb{S}_{+}^{n}, \mathrm{~A} \in \mathbb{R}^{m \times n}$, and $\mathrm{G} \in \mathbb{R}^{m \times p}$ as

$$
\begin{aligned}
& \mathrm{H} \triangleq\left[\begin{array}{cccccc}
Q_{x} & Q_{x u} & 0 & \cdots & & 0 \\
Q_{x u}^{T} & Q_{u} & 0 & \ldots & & 0 \\
0 & 0 & \ddots & \ddots & & \vdots \\
\vdots & \vdots & \ddots & Q_{x} & Q_{x u} & 0 \\
& & & Q_{x u}^{T} & Q_{u} & 0 \\
0 & 0 & \ldots & 0 & 0 & 0
\end{array}\right] \\
& \mathrm{A} \triangleq\left[\begin{array}{ccccccc}
-I & 0 & \ldots & & & \cdots & 0 \\
A & B & -I & 0 & \ldots & & \vdots \\
0 & 0 & A & \ldots & & & \\
\vdots & \vdots & & \ddots & & & 0 \\
\vdots & \vdots & & & A & B & -I \\
0 & \cdots & & & \cdots & 0 & -I
\end{array}\right] \\
& \mathrm{G} \triangleq\left[\begin{array}{cc}
-I & 0 \\
0 & 0 \\
\vdots & \vdots \\
0 & 0 \\
-\hat{A} & -\hat{B}
\end{array}\right], \quad X_{i} \triangleq\left[\begin{array}{c}
x_{0, i} \\
u_{0, i} \\
\vdots \\
u_{N_{i}-1, i} \\
x_{N_{i}, i}
\end{array}\right]
\end{aligned}
$$

For the last subproblem $i=\hat{N}$, the matrices are defined similarly as in (5) but including the cost for $x_{N_{\hat{N}}, \hat{N}}$ in $\mathrm{H}_{\hat{N}}$, removing the last block row in $\mathrm{A}_{\hat{N}}$ and $\mathrm{G}_{\hat{N}}$, and removing the last block column in $\mathrm{G}_{\hat{N}}$ since no terminal constraint is introduced in the last subproblem.

By using the definitions in (5) together with the coupling constraints and the complicating variables, an extended optimization problem can be constructed from (1), given by

$$
\begin{array}{cl}
\min _{\hat{\mathrm{x}}, \hat{\mathrm{u}}, \mathrm{x}} & \sum_{i=0}^{\hat{N}-1} \frac{1}{2} X_{i}^{T} \mathrm{H} X_{i}+\frac{1}{2} X_{\hat{N}}^{T} \mathrm{H}_{\hat{N}} X_{\hat{N}} \\
\text { s.t. } & \mathrm{A} X_{i}=\mathrm{G}\left[\begin{array}{l}
\hat{x}_{i} \\
\hat{u}_{i}
\end{array}\right], i \in \mathbb{Z}_{0, \hat{N}-1} \\
& \mathrm{~A}_{\hat{N}} X_{\hat{N}}=\mathrm{G}_{\hat{N}} \hat{x}_{\hat{N}} \\
& \hat{x}_{0}=\bar{x} \\
& \hat{x}_{i+1}=\hat{A} \hat{x}_{i}+\hat{B} \hat{u}_{i}, i \in \mathbb{Z}_{0, \hat{N}-1} .
\end{array}
$$

The extended problem (6) can be solved by first optimizing over the local variables $X_{i}$ while considering the complicating variables as parameters. By defining the parameters

$$
\theta_{i} \triangleq\left[\begin{array}{l}
\hat{x}_{i} \\
\hat{u}_{i}
\end{array}\right], i \in \mathbb{Z}_{0, \hat{N}-1}, \quad \theta_{\hat{N}} \triangleq \hat{x}_{\hat{N}},
$$

the optimal solution to $X_{i}$ for $i \in \mathbb{Z}_{0, \hat{N}}$ can be computed as a function of the parameter $\theta_{i}$ by solving the equality constrained mp-QP problem

$$
\begin{array}{cl}
\min _{X_{i}} & \frac{1}{2} X_{i}^{T} \mathrm{H} X_{i} \\
\text { s.t. } & \mathrm{A} X_{i}=\mathrm{G} \theta_{i} .
\end{array}
$$

Lemma 1: Consider an mp-QP problem as in (8) with $\mathcal{N}(A) \neq\{0\}$. Let the columns of $V$ form a basis of $\mathcal{N}(\mathrm{A})$. Then $V^{T} \mathrm{H} V \succ 0$ holds.

Proof: Consider a subproblem $i \in \mathbb{Z}_{0, \hat{N}-1}$. Let $\bar{A}$ and $\bar{G}$ denote all the rows in $A$ and $G$ except the last block row. Now consider a problem as in (8), but with the equality constraints $\overline{\mathrm{A}} X_{i}=\overline{\mathrm{G}} \theta_{i}$ instead. This problem is a UFTOC problem in the form (1) where Assumptions 1 and 2 hold, which has a unique solution [28]. Hence, the reduced Hessian $\bar{V}^{T} \mathrm{H} \bar{V}$ is positive definite, where the columns of $\bar{V}$ form a basis for $\mathcal{N}(\overline{\mathrm{A}})$ [29]. Since $\mathcal{N}(\mathrm{A}) \subseteq \mathcal{N}(\overline{\mathrm{A}})$, the result follows. For $i=\hat{N}$ the result follows immediately since it is in the UFTOC problem where Assumptions 1 and 2 hold.

The subproblem (8) is only feasible when $\mathrm{G} \theta_{i} \in \mathcal{R}(\mathrm{A})$ [30]. Hence, depending on the parametrization and the subproblem, the introduction of the terminal constraints might give infeasible subproblems. In order to obtain feasible subproblems in (6) for every choice of the parameters, the parametrizations of the terminal constraints must be chosen such that it is always possible to reach the desired final state from the initial state of the subproblem. Given the initial state, the final state can be computed using the dynamics constraints as

$$
x_{N_{i}, i}=\tilde{\mathrm{A}} \hat{x}_{i}+\tilde{\mathrm{B}} \mathrm{u}_{i}
$$

where

$$
\begin{aligned}
& \tilde{\mathrm{A}} \triangleq A^{N_{i}}, \quad \tilde{\mathrm{D}} \triangleq\left[\begin{array}{llll}
A^{N_{i}-1} & \cdots & A & I
\end{array}\right], \\
& \tilde{\mathrm{B}} \triangleq\left[\begin{array}{llll}
A^{N_{i}-1} B & \cdots & A B & B
\end{array}\right],
\end{aligned}
$$


and $\mathrm{u}_{i}$ is the stacked $u_{t, i}$ for $t \in \mathbb{Z}_{0, N_{i}-1}$. Note that $\tilde{\mathrm{B}}$ is the reachability matrix [31], and $\tilde{D}$ is defined for later purposes. The feasibility of a subproblem can be managed by parametrizing the problem such that the parameter $d_{i}=\hat{A} \hat{x}_{i}+\hat{B} \hat{u}_{i}$ is reachable from $\hat{x}_{i}$. This can be assured by choosing

$$
\hat{A} \triangleq \tilde{\mathrm{A}}, \quad \hat{B} \triangleq \mathcal{T}
$$

where the columns of $\mathcal{T}$ form a basis for $\mathcal{R}(\tilde{B})$. Note that by introducing this parametrization of the subproblem, the problem of choosing parameters in the master problem that satisfy the range constraints $\mathrm{G} \theta_{i} \in \mathcal{R}(\mathrm{A})$ for $i \in \mathbb{Z}_{0, \hat{N}-1}$ to give feasible subproblems is solved already in the subproblems and not in the master problem as in [18], [19]. Note that for a subproblem where $\tilde{B}$ has full row rank, $\hat{A}=0$ and $\hat{B}=I$ are valid choices for the parametrization of the terminal constraint.

Let the notation $\leftrightarrow$ be used to relate a dual variable with its corresponding constraint. Then, define

$$
\begin{aligned}
\lambda_{0, i} & \leftrightarrow-x_{0, i}+\hat{x}_{i}=0, \\
\lambda_{t+1, i} & \leftrightarrow-x_{t+1, i}+A x_{t, i}+B u_{t, i}=0, t \in \mathbb{Z}_{0, N_{i}-1}, \\
\lambda_{t c, i} & \leftrightarrow-x_{N_{i}, i}+\hat{A} \hat{x}_{i}+\hat{B} \hat{u}_{i}=0,
\end{aligned}
$$

as the dual variables for the problem (8), and $\Lambda_{i}$ as

$$
\Lambda_{i} \triangleq\left[\begin{array}{lllll}
\lambda_{0, i}^{T} & \lambda_{1, i}^{T} & \ldots & \lambda_{N_{i}, i}^{T} & \lambda_{t c, i}^{T}
\end{array}\right]^{T} .
$$

The subproblem (8) is a very simple mp-QP problem with parameters $\theta_{i}$ and only equality constraints, with optimal primal and dual solutions given by

$$
\begin{aligned}
X_{i}^{*}\left(\theta_{i}\right) & =K^{x} \theta_{i}, \\
\Lambda_{i}^{*}\left(\theta_{i}\right) & =K^{\lambda} \theta_{i}+\lambda_{i}^{\mathcal{N}},
\end{aligned}
$$

for some $K^{x} \in \mathbb{R}^{n \times p}, K^{\lambda} \in \mathbb{R}^{m \times p}$, and $\lambda_{i}^{\mathcal{N}} \in \mathcal{N}\left(\mathrm{A}^{T}\right)$, [32]. The primal solution is unique since either the reduced Hessian is positive definite according to Lemma 1 , or $\mathcal{N}(A)=\{0\}$. When LICQ holds for the subproblem (8) it follows that $\mathcal{N}\left(\mathrm{A}^{T}\right)=\{0\}$, and hence also the dual solution is unique [32]. Note that LICQ always holds for the last subproblem $i=\hat{N}$. Furthermore, since the simple mp-QP problem (8) is subject to equality constraints only, the solution can be computed cheaply compared to a general mp-QP problem.

The local variables $X_{i}$ can be eliminated from the extended problem (6) by using the parametric solution (14a). The value function of (8) can then be expressed in the parameter $\theta_{i}$ as

$$
\hat{V}\left(\theta_{i}\right) \triangleq \frac{1}{2} \theta_{i}^{T}\left(K^{x}\right)^{T} \mathrm{H} K^{x} \theta_{i}, \quad \hat{V}_{\hat{N}} \triangleq \frac{1}{2} \theta_{\hat{N}}^{T}\left(K_{\hat{N}}^{x}\right)^{T} \mathrm{H}_{\hat{N}} K_{\hat{N}}^{x} \theta_{\hat{N}} .
$$

Lemma 2: Let the Hessian of $\hat{V}\left(\theta_{i}\right)$ be divided into blocks that correspond to $\hat{x}_{i}$ and $\hat{u}_{i}$ as

$$
\hat{Q} \triangleq\left[\begin{array}{cc}
\hat{Q}_{x} & \hat{Q}_{x u} \\
\hat{Q}_{x u}^{T} & \hat{Q}_{u}
\end{array}\right] \triangleq\left(K^{x}\right)^{T} \mathrm{H} K^{x}
$$

Then it holds that $\hat{Q}_{u} \in \mathbb{S}_{++}^{n_{\hat{u}}}$.

Proof: The proof of Lemma 2 is presented in Appendix D.

\section{B. Constructing the master problem}

By eliminating all the local variables $X_{i}$ for $i \in \mathbb{Z}_{0, \hat{N}}$ in (6), a master UFTOC problem with horizon $\hat{N}<N$ is obtained.

Theorem 1: Consider a UFTOC problem $\mathcal{P}(N)$ as defined in (1). Then, this UFTOC problem can be reduced in parallel to a smaller UFTOC problem $\mathcal{P}(\hat{N})$ with $\hat{N}<N$, given by

$$
\begin{aligned}
\min _{\hat{\mathrm{x}}, \hat{\mathrm{u}}} & \sum_{i=0}^{\hat{N}-1} \frac{1}{2}\left[\begin{array}{l}
\hat{x}_{i} \\
\hat{u}_{i}
\end{array}\right]^{T}\left[\begin{array}{cc}
\hat{Q}_{x} & \hat{Q}_{x u} \\
\hat{Q}_{x u}^{T} & \hat{Q}_{u}
\end{array}\right]\left[\begin{array}{l}
\hat{x}_{i} \\
\hat{u}_{i}
\end{array}\right]+\frac{1}{2} \hat{x}_{\hat{N}}^{T} \hat{Q}_{x, \hat{N}} \hat{x}_{\hat{N}} \\
\text { s.t. } & \hat{x}_{0}=\bar{x} \\
& \hat{x}_{i+1}=\hat{A} \hat{x}_{i}+\hat{B} \hat{u}_{i}, i \in \mathbb{Z}_{0, \hat{N}-1},
\end{aligned}
$$

where $\hat{Q} \in \mathbb{S}_{+}^{n_{x}+n_{\hat{u}}}$ and $\hat{Q}_{u} \in \mathbb{S}_{++}^{n_{\hat{u}}}$ are defined in (16), $\hat{A} \in$ $\mathbb{R}^{n_{x} \times n_{x}}$ and $\hat{B} \in \mathbb{R}^{n_{x} \times n_{\hat{u}}}$ are defined in (11), and $n_{\hat{u}} \leq n_{x}$.

Proof: Given a UFTOC problem (1), the construction of the extended problem (6) can be done as described in Section IV-A. All local variables can be eliminated by solving the subproblems (8) parametrically as functions of the complicating variables, and substituting the parametric solution into the extended problem (6). Then, using the definitions in (16) it follows that the extended problem (6) is reduced to (17). $\hat{A}$ and $\hat{B}$ are chosen according to (11), and the parametrization is introduced such that $n_{\hat{u}} \leq n_{x}$. Positive semidefiniteness of $\hat{Q}$ follows by construction, and $\hat{Q}_{u} \in \mathbb{S}_{++}^{n_{\hat{u}}}$ follows from Lemma 2. Furthermore, since all subproblems can be solved parametrically independently of each other, it is possible to reduce the UFTOC problem (1) to (17) in parallel.

Let the dual variables for the master UFTOC problem in (17) be defined as

$$
\begin{aligned}
& \hat{\lambda}_{0} \leftrightarrow-\hat{x}_{0}+\bar{x}=0, \\
& \hat{\lambda}_{i+1} \leftrightarrow-\hat{x}_{i+1}+\hat{A} \hat{x}_{i}+\hat{B} \hat{u}_{i}=0, i \in \mathbb{Z}_{0, \hat{N}-1} .
\end{aligned}
$$

Then, the primal and dual solution given by $\hat{x}_{i}^{*}$ for $i \in \mathbb{Z}_{0, \hat{N}}$, $\hat{u}_{i}^{*}$ for $i \in \mathbb{Z}_{0, \hat{N}-1}$ and $\hat{\lambda}_{i}^{*}$ for $i \in \mathbb{Z}_{0, \hat{N}}$ is unique since LICQ and Assumptions 1 and 2 hold for the master problem. The solution can be computed using any method that is applicable to UFTOC problems in the form (17).

\section{Computing the solution}

The solutions to the subproblems (8) can be computed using the optimal values of the parameters $\theta_{i}$ from the solution to the master problem (17) using (14). When LICQ holds, the primal and dual solution $X_{i}^{*}$ and $\Lambda_{i}^{*}$ can be computed uniquely from the optimal parameters $\theta_{i}^{*}$ as in (14) but with $\lambda_{i}^{\mathcal{N}}=0$.

However, the additional terminal constraint in a subproblem (8) might result in violation of LICQ for the subproblem even though this is not the case in the original UFTOC problem (1). Violation of LICQ is known as primal degeneracy and the dual variables (14b) for a primally degenerate problem are non-unique [32]. Here it will be shown how to choose dual variables in the subproblems that satisfy the KKT optimality conditions of the original UFTOC problem (1), even in the case with non-unique dual variables in the subproblems.

Lemma 3: The nullspace of $A^{T}$ is given by

$$
\mathcal{N}\left(\mathrm{A}^{T}\right) \triangleq\left\{z \in \mathbb{R}^{m} \mid z=Z w, \text { with } w \in \mathcal{N}\left(\tilde{\mathrm{B}}^{T}\right)\right\}
$$


where

$$
Z \triangleq\left[\begin{array}{lll}
-\tilde{\mathrm{A}} & -\tilde{\mathrm{D}} & I
\end{array}\right]^{T},
$$

and $\tilde{A}, \tilde{D}$, and $\tilde{B}$ are defined in (10).

Proof: For the proof of Lemma 3, see Appendix F.

How to compute the solution to the original UFTOC problem from $X_{i}^{*}$ and $\Lambda_{i}^{*}$ for $i \in \mathbb{Z}_{0, \hat{N}}$ is presented in Theorem 2 .

Theorem 2: Let Assumptions 1 and 2 hold for the UFTOC problem in (1), and let the corresponding extended problem (6) be constructed as described in Section IV-A. Furthermore, let $\hat{x}^{*}, \hat{u}^{*}$ and $\hat{\lambda}^{*}$ be the solution to (17). Then, the primal and dual solution to (1) can be computed as

$$
\mathbf{x}^{*}=\left[\begin{array}{c}
x_{0,0}^{*} \\
\vdots \\
x_{N_{i}-1, i}^{*} \\
x_{0, i+1}^{*} \\
\vdots \\
x_{N_{\hat{N}}, \hat{N}}^{*}
\end{array}\right], \quad \mathbf{u}^{*}=\left[\begin{array}{c}
u_{0,0}^{*} \\
\vdots \\
u_{N_{i}-1, i}^{*} \\
u_{0, i+1}^{*} \\
\vdots \\
u_{N_{\hat{N}}-1, \hat{N}}^{*}
\end{array}\right], \quad \lambda^{*}=\left[\begin{array}{c}
\lambda_{0,0}^{*} \\
\vdots \\
\lambda_{N_{i}-1, i}^{*} \\
\lambda_{0, i+1}^{*} \\
\vdots \\
\lambda_{N_{\hat{N}}, \hat{N}}^{*}
\end{array}\right],
$$

where $x_{t, i}^{*}$ and $u_{t, i}^{*}$ are computed as in (14a) for $i \in \mathbb{Z}_{0, \hat{N}}$, and $\Lambda_{i}^{*}$ are computed as in (14b) with $\lambda_{i}^{\mathcal{N}}=0$ when LICQ holds. When LICQ does not hold, $\Lambda_{i}^{*}$ is computed as

$$
\Lambda_{i}^{*}=K^{\lambda} \theta_{i}^{*}-Z\left(\xi_{t c, i}^{*}+\hat{\lambda}_{i+1}^{*}\right),
$$

where $\theta_{i}$ is defined as in (7), $Z$ is given in Lemma 3, and

$$
\xi_{t c, i}^{*} \triangleq\left[\begin{array}{llll}
0 & \cdots & 0 & I_{n_{x}}
\end{array}\right] K^{\lambda}\left[\begin{array}{c}
\hat{x}_{i}^{*} \\
\hat{u}_{i}^{*}
\end{array}\right] .
$$

Proof: The proof of Theorem 2 is given in Appendix E.

According to Theorem 2, the solution to the original UFTOC problem (1) can be computed from the parametric solutions to the subproblems (8) and the solution to the master problem (17). Since all subproblems can be solved independently of each other when $\hat{x}^{*}, \hat{u}^{*}$ and $\hat{\lambda}^{*}$ are given, it is possible to compute the solution to the $\hat{N}+1$ subproblems, and hence also to the original UFTOC problem, in parallel.

\section{Parallel Partial Condensing AND REDUCTION APPROACH}

In [24] partial condensing was introduced as a way to lower the computation time for solving MPC problems by enabling condensing strategies that are in between the sparse MPC formulation and the fully condensed MPC formulation. As was remarked in that work, the ideas from partial condensing can also be used in a parallel setting, where for example the condensing of each block can be performed in parallel and the sizes of the blocks determine the corresponding workloads. In this section it will be shown how to exploit, and extend, the ideas from partial condensing in order to reduce a UFTOC problem in parallel to a smaller master problem in the same UFTOC form but with shorter prediction horizon and possibly fewer control inputs. Furthermore, it will also be shown how the parallel Riccati recursion algorithm presented in [25] can be included in this framework. Here, this is done in a similar way as [14], but in the algorithm presented here the reduced master problem remains in the UFTOC form, which facilitates the recursive solution of smaller UFTOC problems. It will also be shown how to compute the Riccati factorization in Algorithm 1 in parallel, which is not part of the work in [14].

The reduction of the original UFTOC problem $\mathcal{P}(N)$ to the master UFTOC problem $\mathcal{P}(\hat{N})$ will be done in two main steps:

- Condense the subproblems: Eliminate states by using the corresponding dynamics constraints. This is similar to what is done when re-writing the UFTOC problem into its condensed QP form as is shown in for example [28], [33], and [24]. The different condensing techniques that are used here are presented in Section V-B.

- Reduce the subproblems: Reduce the size of the total control input dimension over the horizon in the subproblem. Depending on the subproblem, it is not always possible to do this, but it will be described in Section V-C when, and how, this can be done.

In this section, it will be described how to perform these steps in parallel, but first the decomposition of the UFTOC problem $\mathcal{P}(N)$ into subproblems is presented.

\section{A. Decomposition into subproblems}

The decomposition of the original UFTOC problem (1) can be done similarly as in Section IV. Let $N_{i}, t_{0}$ and $t_{i}$ be defined as before, and define $t_{\hat{N}+1} \triangleq N$. Furthermore, for presentational brevity, let $\hat{x}_{i} \triangleq x_{t_{i}}$ denote the state at time $t_{i}$ and define the local variables $\mathrm{x}_{i}$ and $\mathrm{u}_{i}$ for $i \in \mathbb{Z}_{0, \hat{N}}$ as

$\mathrm{x}_{i} \triangleq\left[\begin{array}{lll}x_{t_{i}+1}^{T} & \ldots & x_{t_{i}+N_{i}-1}^{T}\end{array}\right]^{T}, \quad \mathbf{u}_{i} \triangleq\left[\begin{array}{lll}u_{t_{i}}^{T} & \ldots & u_{t_{i}+N_{i}-1}^{T}\end{array}\right]^{T}$

Note that the indexing starts at different values for $\mathrm{x}_{i}$ and $\mathrm{u}_{i}$. Then, the UFTOC problem (1) is equivalent to

$$
\begin{aligned}
\min _{\hat{\mathrm{x}}, \mathrm{x}, \mathrm{u}} & \sum_{i=0}^{\hat{N}} \frac{1}{2}\left[\begin{array}{c}
\hat{x}_{i} \\
x_{i} \\
\mathrm{u}_{i}
\end{array}\right]^{T}\left[\begin{array}{ccc}
Q_{x} & 0 & \mathrm{Q}_{x \mathrm{u}} \\
0 & \mathrm{Q}_{\mathrm{x}} & \mathrm{Q}_{\mathrm{xu}} \\
\mathrm{Q}_{x \mathrm{u}}^{T} & \mathrm{Q}_{\mathrm{xu}}^{T} & \mathrm{Q}_{\mathrm{u}}
\end{array}\right]\left[\begin{array}{c}
\hat{x}_{i} \\
\mathrm{x}_{i} \\
\mathrm{u}_{i}
\end{array}\right]+ \\
& \frac{1}{2} \hat{x}_{\hat{N}+1}^{T} Q_{x, N} \hat{x}_{\hat{N}+1} \\
\text { s.t. } & \hat{x}_{0}=\bar{x} \\
& \mathrm{Ax}_{i}=\mathrm{A}_{0} \hat{x}_{i}+\mathrm{Bu}_{i}, i \in \mathbb{Z}_{0, \hat{N}} \\
& \hat{x}_{i+1}=\mathrm{A}_{N} \mathrm{x}_{i}+\mathrm{B}_{N} \mathrm{u}_{i}, i \in \mathbb{Z}_{0, \hat{N}},
\end{aligned}
$$

where the matrices are defined in Appendix A. Note that $A$ is an invertible matrix by definition and that no extra variables or constraints have been added to the original UFTOC problem to form the equivalent (25) as was done in Section IV.

Instead of introducing complicating variables and constraints as in Section IV, the original UFTOC problem will now be decomposed into several subproblems using the cost-to-go function from dynamic programming, see for example [34]. To do this, let $J\left(x_{t}\right)$ denote the cost-to-go function at state $x_{t}$ for the UFTOC problem (1), which in this case is a convex quadratic function in $x_{t}$ given by

$$
J\left(x_{t}\right) \triangleq \frac{1}{2} x_{t}^{T} P_{t} x_{t}, \quad P_{t} \in \mathbb{S}_{+}^{n_{x}} .
$$

Furthermore, define $\bar{x}_{i} \triangleq x_{t_{i}}^{*}$ where $x_{t_{i}}^{*}$ is part of the optimal solution to the problem (1). Assume, for the moment, that $\bar{x}_{i}$ 
and $J\left(\hat{x}_{i+1}\right)$ are known for some $i \in \mathbb{Z}_{0, \hat{N}}$. Then it is possible to exploit the principle of optimality [34] and the structure of the equality constraints in the UFTOC problem (1) to compute the optimal solution in the interval $t_{i} \leq t \leq t_{i+1}$. This is done by solving the smaller UFTOC problem

$$
\min _{\hat{x}_{i}, \mathrm{x}_{i}, \mathrm{u}_{i}, \hat{x}_{i+1}} \frac{1}{2}\left[\begin{array}{c}
\hat{x}_{i} \\
\mathrm{x}_{i} \\
\mathrm{u}_{i}
\end{array}\right]^{T}\left[\begin{array}{ccc}
Q_{x} & 0 & \mathrm{Q}_{x \mathrm{u}} \\
0 & \mathrm{Q}_{\mathrm{x}} & \mathrm{Q}_{\mathrm{xu}} \\
\mathrm{Q}_{x \mathrm{u}}^{T} & \mathrm{Q}_{\mathrm{xu}}^{T} & \mathrm{Q}_{\mathrm{u}}
\end{array}\right]\left[\begin{array}{c}
\hat{x}_{i} \\
\mathrm{x}_{i} \\
\mathrm{u}_{i}
\end{array}\right]+\underbrace{\frac{1}{2} \hat{x}_{i+1}^{T} \hat{P}_{i+1} \hat{x}_{i+1}}_{=J\left(\hat{x}_{i+1}\right)}
$$$$
\text { s.t. } \quad \hat{x}_{i}=\bar{x}_{i}
$$$$
\mathrm{Ax}_{i}=\mathrm{A}_{0} \hat{x}_{i}+\mathrm{Bu}_{i}
$$$$
\hat{x}_{i+1}=\mathrm{A}_{N} \mathrm{x}_{i}+\mathrm{B}_{N} \mathrm{u}_{i} \text {. }
$$

Here, the notation $\hat{P}_{i+1} \triangleq P_{t_{i+1}}$ is used for brevity. Hence, if $\bar{x}_{i}$ and $J\left(\hat{x}_{i+1}\right)$ are known for all $i \in \mathbb{Z}_{0, \hat{N}}$ it is possible to solve the original UFTOC problem (1) by computing the solution in each interval $t_{i} \leq t \leq t_{i+1}$, which is done by solving the $\hat{N}+1$ corresponding subproblems in the form (27).

Remark 1: For the last subproblem $i=\hat{N}, J\left(\hat{x}_{\hat{N}+1}\right)$ is known and defined as $J\left(\hat{x}_{\hat{N}+1}\right)=\frac{1}{2} \hat{x}_{\hat{N}+1}^{T} Q_{x, N} \hat{x}_{\hat{N}+1}$.

\section{B. Condensing a subproblem}

The optimal solutions $\bar{x}_{i}$ and the cost-to-go functions $J\left(\hat{x}_{i+1}\right)$ are not available prior to solving the problem (except for $J\left(\hat{x}_{\hat{N}+1}\right)$ as mentioned in Remark 1). However, it is possible to eliminate local state variables from the subproblem (27), which is here referred to as condensing the subproblem. Here, it will be shown how to condense the subproblem in two different, but conceptually similar, ways. The first condensing technique is the one used in for example [28], [33], and [24] and will be referred to as regular condensing, whereas the other is based on the ideas from [25] where the Riccati recursion is used to obtain the condensed subproblem.

1) Regular condensing of a subproblem: By using the dynamics constraints in the UFTOC problem (27) it is possible to eliminate the state variables by describing them as a function of the initial state and the control inputs over the full horizon of the subproblem. For later purposes, the final state is kept as a variable in the condensed subproblem.

Lemma 4 (Regular condensing): Consider the QP problem (27) with invertible $A$, convex objective function and $Q_{u} \succ 0$.

Then, eliminating the variables $\mathrm{x}_{i}$ using the equality constraints $\mathrm{Ax}_{i}=\mathrm{A}_{0} \hat{x}_{i}+\mathrm{Bu}_{i}$ results in the $\mathrm{QP}$ problem

$$
\begin{aligned}
\min _{\hat{x}_{i}, \mathrm{u}_{i}, \hat{x}_{i+1}} & \frac{1}{2}\left[\begin{array}{c}
\hat{x}_{i} \\
\mathrm{u}_{i}
\end{array}\right]^{T}\left[\begin{array}{cc}
\tilde{Q}_{x} & \tilde{Q}_{x \mathrm{u}} \\
\tilde{Q}_{x \mathrm{u}}^{T} & \tilde{Q}_{\mathrm{u}}
\end{array}\right]\left[\begin{array}{c}
\hat{x}_{i} \\
\mathrm{u}_{i}
\end{array}\right]+\frac{1}{2} \hat{x}_{i+1}^{T} \hat{P}_{i+1} \hat{x}_{i+1} \\
\text { s.t. } & \hat{x}_{0}=\bar{x}_{i} \\
& \hat{x}_{i+1}=\tilde{A} \hat{x}_{i}+\tilde{\mathrm{B}} \mathrm{u}_{i},
\end{aligned}
$$

where the matrices are defined as in (60) in Appendix B, the objective function is convex, and $\tilde{Q}_{\mathrm{u}} \succ 0$.

Furthermore, the eliminated variables $\mathrm{x}_{i}$ and the dual variables $\lambda_{i} \leftrightarrow-\mathrm{Ax}_{i}+\mathrm{A}_{0} \hat{x}_{i}+\mathrm{Bu}_{i}=0$ can be computed as

$$
\begin{aligned}
\mathrm{x}_{i} & =\mathrm{A}^{-1} \mathrm{~A}_{0} \hat{x}_{i}+\mathrm{A}^{-1} \mathrm{Bu}_{i}, \\
\lambda_{i} & =\mathrm{A}^{-T}\left(\mathrm{Q}_{\mathrm{x}} \mathrm{x}_{i}+\mathrm{Q}_{\mathrm{xu}} \mathrm{u}_{i}\right) .
\end{aligned}
$$

Proof: The proof of Lemma 4 is given in the Appendix G.

The condensing of all subproblems using Lemma 4 can be interpreted as partially condensing the UFTOC problem (1) as in [24]. The condensed problem (28) can be interpreted as a UFTOC problem $\mathcal{P}(1)$ with $m \triangleq N_{i} n_{u}$ control inputs.

2) Condensing using the Riccati recursion: First, an assumption that will be important later when the Riccati-based condensing technique is used is presented next:

Assumption 3: At least one of the following properties holds

(i) $Q_{u} \in \mathbb{S}_{++}^{n_{u}}$,

(ii) $Q_{u}=B \in \mathbb{S}_{+}^{n_{x}}$.

When using the Riccati-based approach to condense the subproblem, Assumption 3 is used instead of Assumption 2 for the UFTOC problem (1). Even though the original UFTOC problem that is solved satisfies Assumption 2, being able to handle UFTOC problems that only satisfy this relaxed assumption will be important in Section VI when UFTOC problems are solved recursively.

The Riccati-based condensing approach is based on a change of variables of $u_{t, i}$ which is obtained by computing the Riccati factorization for a preliminary choice of $J\left(\hat{x}_{i+1}\right)$. Although this condensation of the subproblem is conceptually the same as the regular one, it will be shown that it results in a condensed subproblem with a structure that can facilitate more efficient computations and communications.

To compute this change of variables, a preliminary feedback is computed by computing the Riccati factorization for the UFTOC problem (27) using Algorithm 1 for the preliminary choice $P_{i+1}=0$. However, since the cost-to-go function

$$
J\left(\hat{x}_{i+1}\right)=\frac{1}{2} \hat{x}_{i+1}^{T} \hat{P}_{i+1} \hat{x}_{i+1},
$$

might not be zero, it is necessary to capture the effect from the cost-to-go function on the solution to the subproblem. Let $\bar{u}_{t} \in \mathbb{R}^{n_{u}}$ be the contribution of the non-zero $J\left(\hat{x}_{i+1}\right)$ on $u_{t}$, and let the subindex " 0 " denote a variable associated with the preliminary factorization. Then $u_{t}$ can be expressed as

$$
u_{t}=K_{0, t+1} x_{t}+\bar{u}_{t}, t \in \mathbb{Z}_{t_{i}, t_{i}+N_{i}-1}
$$

which can be interpreted as a change of variables from $u_{t}$ to $\bar{u}_{t}$. Note that $\bar{u}_{t}$ is a full $n_{u}$ vector and hence there is no loss of generality when using the change of variables (31). By using (31) a condensed UFTOC problem similar to (28) but with a different structure in the objective function can be obtained. This condensing approach is described in Lemma 5.

Lemma 5 (Condensing using the Riccati recursion): Consider a UFTOC problem in the form (27) where Assumptions 1 and 3 hold, and assume that Algorithm 1 has been computed for $\hat{P}_{i+1}=0$, giving $P_{0, t}$ and $K_{0, t+1}$. Then, by using (31), the problem $(27)$ can be condensed to a UFTOC problem $\mathcal{P}(1)$

$$
\begin{aligned}
& \min _{\hat{x}_{i}, \overline{\mathrm{u}}_{i}, \hat{x}_{i+1}} \frac{1}{2} \hat{x}_{i}^{T} P_{0, t_{i}} \hat{x}_{i}+\frac{1}{2} \overline{\mathrm{u}}_{i}^{T} \overline{\mathrm{Q}}_{\overline{\mathrm{u}}} \overline{\mathrm{u}}_{i}+\frac{1}{2} \hat{x}_{i+1}^{T} \hat{P}_{i+1} \hat{x}_{i+1} \\
& \text { s.t. } \hat{x}_{i}=\bar{x}_{i} \\
& \hat{x}_{i+1}=\hat{A} \hat{x}_{i}+\mathrm{Su}_{i} \text {, }
\end{aligned}
$$


with $\overline{\mathrm{u}}_{i} \in \mathbb{R}^{m}$ and

$$
\begin{aligned}
& \overline{\mathrm{Q}}_{\overline{\mathrm{u}}} \triangleq\left[\begin{array}{lll}
G_{0, t_{i}+1} & & \\
& \ddots & \\
& & G_{0, t_{i}+N_{i}}
\end{array}\right] \in \mathbb{S}_{+}^{m}, \\
& \hat{A} \triangleq \prod_{t=t_{i}}^{t_{i}+N_{i}-1}\left(A+B K_{0, t+1}\right) \text {, } \\
& \mathrm{S} \triangleq\left[\begin{array}{llll}
\prod_{t=t_{i}+1}^{t_{i}+N_{i}-1}\left(A+B K_{0, t+1}\right) B & \ldots & B
\end{array}\right] .
\end{aligned}
$$

If Assumption 3(i) holds then $\bar{Q}_{\bar{u}} \in \mathbb{S}_{++}^{m}$.

Furthermore, by using the notation $\hat{\lambda}_{i+1} \triangleq \lambda_{t_{i+1}}$, the eliminated states $x_{t}$ and dual variables $\lambda_{t}$ for $t \in \mathbb{Z}_{t_{i}+1, t_{i}+N_{i}-1}$ can be computed from

$$
\begin{aligned}
x_{t+1} & =A x_{t}+B u_{t}, t \in \mathbb{Z}_{t_{i}, t_{i}+N_{i}-2}, \\
\lambda_{t} & =P_{0, t} x_{t}+\left(\prod_{\tau=t}^{t_{i}+N_{i}-1}\left(A+B K_{0, \tau+1}\right)\right)^{T} \hat{\lambda}_{i+1} .
\end{aligned}
$$

Proof: The proof of Lemma 5 is given in Appendix H.

By using Lemma 5, it is possible to condense the UFTOC problem (27) into the problem (32), with the matrices defined as in (33). This condensed problem is in the UFTOC form $\mathcal{P}(1)$ with $m$ control inputs, which is in the same form as the condensed problem (28). However, (32) possibly has $\bar{Q}_{\bar{u}} \in \mathbb{S}_{+}^{m}$ since Assumption 3 is used instead of Assumption 2, and the cross terms between $\hat{x}_{i}$ and $\overline{\mathrm{u}}_{i}$ in the objective function in (32) are eliminated when using the change of variables in (31). Furthermore, the quadratic cost matrix $\bar{Q}_{\bar{u}}$ is block diagonal as can be seen in (33a). It will be shown that these properties are important from both a computation and communication point of view. Note that $S$ is the reachability matrix for the subproblem (27) when using the change of variables (31). Hence, it is closely related to the reachability matrix $\tilde{B}$ in (10b) for the subproblems in the parametric programming approach.

\section{Reducing the control input dimension in a subproblem}

When using any of the two condensing techniques described in Section V-B, the resulting condensed subproblems (28) and (32) are both in the same UFTOC form $\mathcal{P}(1)$ with $m$ number of control inputs, but with different problem matrices. Whenever colrank $\tilde{\mathrm{B}}<m$ in (28) or colrank $\mathrm{S}<m$ in (32), respectively, it is possible to reduce the number of control inputs to obtain a subproblem with fewer variables. This case corresponds to subproblems which after the condensing process become over-actuated, and the reduction of the control input dimension can be interpreted as performing control allocation. It will be shown how to reduce the control input dimension in the subproblem in three different ways, where two of them are tailored for subproblems that are condensed using the Riccati recursion approach as in Lemma 5.

1) Regular reduction: For the first reduction approach the following lemma will be used:

Lemma 6 (Reduce control input dimension): Consider a UFTOC problem in the form in (28) with a convex objective function, $\tilde{Q}_{\mathrm{u}} \succ 0$, and where $n_{\hat{u}} \triangleq$ colrank $\tilde{\mathrm{B}}<m$ holds.
Then, this problem can be reduced to the QP problem

$$
\begin{aligned}
\min _{\hat{x}_{i}, \hat{u}_{i}, \hat{x}_{i+1}} & \frac{1}{2}\left[\begin{array}{c}
\hat{x}_{i} \\
\hat{u}_{i}
\end{array}\right]^{T}\left[\begin{array}{cc}
\hat{Q}_{x} & \hat{Q}_{x u} \\
\hat{Q}_{x u}^{T} & \hat{Q}_{u}
\end{array}\right]\left[\begin{array}{c}
\hat{x}_{i} \\
\hat{u}_{i}
\end{array}\right]+\frac{1}{2} \hat{x}_{i+1}^{T} \hat{P}_{i+1} \hat{x}_{i+1} \\
\text { s.t. } & \hat{x}_{i}=\bar{x}_{i} \\
& \hat{x}_{i+1}=\hat{A} \hat{x}_{i}+\hat{B} \hat{u}_{i},
\end{aligned}
$$

where the objective function is convex, $\hat{u}_{i} \in \mathbb{R}^{n_{\hat{u}}}$ and $\hat{Q}_{u} \in$ $\mathbb{S}_{++}^{n_{\hat{u}}}$. The matrices are defined as in (61) in Appendix C.

The variable $\mathrm{u}_{i}$ can be recovered from

$\mathrm{u}_{i}=\left(I-V\left(V^{T} \hat{Q}_{u} V\right)^{-1} V^{T} \hat{Q}_{u}\right) U \hat{u}_{i}-V\left(V^{T} \hat{Q}_{u} V\right)^{-1} V^{T} \hat{Q}_{x u}^{T} \bar{x}_{i}$,

where $U$ and $V$ are defined as in the proof in Appendix I.

Proof: The proof of Lemma 6 is given in Appendix I.

Remark 2: When $\bar{Q}_{\bar{u}} \in \mathbb{S}_{++}^{m}$, the UFTOC problem (32) is in the same form as (28) but with other values of the matrices and can hence be reduced using Lemma 6.

From Lemma 6 and Remark 2 it follows that the subproblems in (28) with colrank $\tilde{\mathrm{B}}<m$, and in (32) with colrank $\mathrm{S}<m$ and $\overline{\mathrm{Q}}_{\overline{\mathrm{u}}} \in \mathbb{S}_{++}^{m}$, can be reduced to a UFTOC problem with $n_{\hat{u}}=$ colrank $\hat{B}=$ colrank $\tilde{\mathrm{B}}=$ colrank $\mathrm{S}$ control inputs. Note that $n_{\hat{u}} \leq n_{x}$ does always hold. For (32) it follows from the definition of the problem and Lemma 6 that $\hat{Q}_{x u}=0$ due to the change of variables (31).

For the case with colrank $\tilde{\mathrm{B}}_{i}=m$ in (28), and colrank $\mathrm{S}_{i}=$ $m$ and $\bar{Q}_{\bar{u}} \in \mathbb{S}_{++}^{m_{i}}$ in (32), the problems are already in the form in (35) and cannot be reduced further.

For the last subproblem $i=\hat{N}$ the cost-to-go function is always known according to Remark 1 . Hence, by computing the Riccati factorization for the problem $\mathcal{P}(1)$ in $(28), J\left(\hat{x}_{\hat{N}}\right)$ and the solution are computed from $\hat{x}_{\hat{N}}$ as

$$
\begin{aligned}
J\left(\hat{x}_{\hat{N}}\right) & =\frac{1}{2} \hat{x}_{\hat{N}}^{T} \hat{P}_{\hat{N}} \hat{x}_{\hat{N}}, \\
\mathrm{u}_{\hat{N}} & =\mathrm{K}_{\hat{N}} \hat{x}_{\hat{N}}, \quad x_{N}=\left(\tilde{A}+\tilde{\mathrm{BK}}_{\hat{N}}\right) \hat{x}_{\hat{N}}, \\
\hat{\lambda}_{\hat{N}} & =\hat{P}_{\hat{N}} \hat{x}_{\hat{N}}, \quad \lambda_{N}=\hat{P}_{\hat{N}+1} \hat{x}_{\hat{N}+1}=Q_{x, N} x_{N} .
\end{aligned}
$$

The solution for the last subproblem when the Riccati based condensing technique is used can be derived analogously.

2) Tailored reduction of the subproblems: The second way of reducing the control input dimension in a subproblem (32), which also works when $\bar{Q}_{\bar{u}} \in \mathbb{S}_{+}^{m}$ due to Assumption 3, is presented next. This approach avoids computing the orthonormal basis $V$ which is required in Lemma 6 . How to compute the Riccati factorization when $G_{t+1}$ is singular due to Assumption 3 is shown in for example [5], [22]. To derive this alternative reduction strategy, Lemma 7 will be used.

Lemma 7: Let $Q_{u}=B \in \mathbb{S}_{+}^{n_{x}}$. Then $\mathcal{N}\left(G_{t+1}\right)=$ $\mathcal{N}\left(Q_{u}\right)=\mathcal{N}(B)$, where $G_{t+1} \in \mathbb{S}_{+}^{n_{x}}$ is defined similarly as in Algorithm 1.

Proof: The lemma follows since $Q_{u}=B$ and $G_{t+1}=$ $Q_{u}+B^{T} P_{t+1} B$ holds for all $t$ such that $\mathcal{N}\left(G_{t+1}\right) \backslash 0 \neq \emptyset$.

The condensed subproblem (32) does not have any crossterms between $\hat{x}_{i}$ and $\overline{\mathrm{u}}_{i}$, which will be exploited here to derive an alternative reduction technique. The solution to the 
condensed problem (32) is obtained by computing the solution to its corresponding KKT system

$$
\left[\begin{array}{ccccc}
0 & -I & 0 & 0 & 0 \\
-I & P_{0, t_{i}} & 0 & \hat{A}^{T} & 0 \\
0 & 0 & \overline{\mathrm{Q}}_{\overline{\mathrm{u}}} & \mathrm{S}^{T} & 0 \\
0 & \hat{A} & \mathrm{~S} & 0 & -I \\
0 & 0 & 0 & -I & \hat{P}_{i+1}
\end{array}\right]\left[\begin{array}{c}
\hat{\lambda}_{i} \\
\hat{x}_{i} \\
\overline{\mathrm{u}}_{i} \\
\hat{\lambda}_{i+1} \\
\hat{x}_{i+1}
\end{array}\right]=\left[\begin{array}{c}
-\bar{x}_{i} \\
0 \\
0 \\
0 \\
0
\end{array}\right]
$$

For the case with $n_{\hat{u}}=$ colrank $\mathrm{S}<m$ it is possible to reduce the size of the system of equations (38) into a smaller one with the same symmetric KKT structure. This will be done by manipulating the KKT matrix (38) in several steps. To start, let $[U V]$ be an orthogonal matrix where the columns of $U \in \mathbb{R}^{m \times n_{\hat{u}}}$ form an orthonormal basis for $\mathcal{R}\left(\mathrm{S}^{T}\right)$ and the columns of $V \in \mathbb{R}^{m \times\left(m-n_{\hat{u}}\right)}$ form an orthonormal basis for $\mathcal{N}(\mathrm{S})$, giving $\mathbb{R}^{m}=U \oplus V$ [30]. Then, by multiplying the third block row in the KKT system (38) with $[U V]^{T}$ from the left, the system of equations can equivalently be written

$$
\left[\begin{array}{ccccc}
0 & -I & 0 & 0 & 0 \\
-I & P_{0, t_{i}} & 0 & \hat{A}^{T} & 0 \\
0 & 0 & U^{T} \overline{\mathrm{Q}}_{\overline{\mathrm{u}}} & U^{T} \mathrm{~S}^{T} & 0 \\
0 & 0 & V^{T} \overline{\mathrm{Q}}_{\overline{\mathrm{u}}} & 0 & 0 \\
0 & \hat{A} & \mathrm{~S} & 0 & -I \\
0 & 0 & 0 & -I & \hat{P}_{i+1}
\end{array}\right]\left[\begin{array}{c}
\hat{\lambda}_{i} \\
\hat{x}_{i} \\
\overline{\mathrm{u}}_{i} \\
\hat{\lambda}_{i+1} \\
\hat{x}_{i+1}
\end{array}\right]=\left[\begin{array}{c}
-\bar{x}_{i} \\
0 \\
0 \\
0 \\
0 \\
0
\end{array}\right],
$$

where $V^{T} \mathrm{~S}^{T}=0$ in the $(4,4)$-block follows from the definition of $V$. The fourth block equation in (39) states that $V^{T} \overline{\mathrm{Q}}_{\overline{\mathrm{u}}} \overline{\mathrm{u}}_{i}=0$ must hold. By exploiting the orthogonality of $\left[\begin{array}{ll}U & V\end{array}\right]$ using the property $V^{T} U=0$, it follows that the equation $V^{T} \bar{Q}_{\overline{\mathrm{u}}} \overline{\mathrm{u}}_{i}=0$ holds whenever

$$
\overline{\mathrm{Q}}_{\overline{\mathrm{u}}} \overline{\mathrm{u}}_{i}=U \hat{u}_{i} \Longleftrightarrow \overline{\mathrm{u}}_{i}=\overline{\mathrm{Q}}_{\overline{\mathrm{u}}}^{\dagger} U \hat{u}_{i}+\left(I-\overline{\mathrm{Q}}_{\overline{\mathrm{u}}}^{\dagger} \overline{\mathrm{Q}}_{\overline{\mathrm{u}}}\right) \hat{z}_{i},
$$

where $\overline{\mathrm{Q}}_{\overline{\mathrm{u}}}^{\dagger}$ is the Moore-Penrose pseudo-inverse of $\overline{\mathrm{Q}}_{\overline{\mathrm{u}}}$ and $\hat{z}_{i} \in$ $\mathbb{R}^{m}$ is an arbitrary vector [35]. From Assumption 3, Lemma 7 and the definitions of $\bar{Q}_{\bar{u}}$ and $S$ it follows that whenever $\bar{Q}_{\bar{u}}$ is singular, $\mathcal{N}\left(\overline{\mathrm{Q}}_{\overline{\mathrm{u}}}\right) \subseteq \mathcal{N}(\mathrm{S})$ holds. Hence, also $\mathcal{R}\left(\mathrm{S}^{T}\right) \subseteq$ $\mathcal{R}\left(\bar{Q}_{\bar{u}}\right)$ holds. Furthermore, since the columns of $U$ form a basis for $\mathcal{R}\left(\mathrm{S}^{T}\right) \subseteq \mathcal{R}\left(\overline{\mathrm{Q}}_{\overline{\mathrm{u}}}\right)$, it implies that

$$
\overline{\mathrm{Q}}_{\overline{\mathrm{u}}} \overline{\mathrm{Q}}_{\overline{\mathrm{u}}}^{\dagger} U=U, \quad \mathrm{~S}=\mathrm{S} \overline{\mathrm{Q}}_{\overline{\mathrm{u}}}^{\dagger} \overline{\mathrm{Q}}_{\overline{\mathrm{u}}},
$$

hold [35]. By using (40) to eliminate $\overline{\mathrm{u}}_{i}$ in (39), together with (41) and $U^{T} U=I$ gives the equivalent system of equations

$$
\left[\begin{array}{ccccc}
0 & -I & 0 & 0 & 0 \\
-I & P_{0, t_{i}} & 0 & \hat{A}^{T} & 0 \\
0 & 0 & I & U^{T} \mathrm{~S}^{T} & 0 \\
0 & \hat{A} & \mathrm{~S} \bar{Q}_{\overline{\mathrm{u}}}^{\dagger} U & 0 & -I \\
0 & 0 & 0 & -I & \hat{P}_{i+1}
\end{array}\right]\left[\begin{array}{c}
\hat{\lambda}_{i} \\
\hat{x}_{i} \\
\hat{u}_{i} \\
\hat{\lambda}_{i+1} \\
\hat{x}_{i+1}
\end{array}\right]=\left[\begin{array}{c}
-\bar{x}_{i} \\
0 \\
0 \\
0 \\
0
\end{array}\right] .
$$

Finally, to obtain a system of equations which has the symmetric KKT structure, the third block row in (42) is multiplied with $U^{T} \overline{\mathrm{Q}}_{\overline{\mathrm{u}}}^{\dagger} U \in \mathbb{S}_{++}^{n_{\hat{u}}}$ from the left. By also noting that
$U U^{T} \mathrm{~S}^{T}=\mathrm{S}^{T}$ follows from the definition of $U$, the system of equations (42) can equivalently be written as

$$
\left[\begin{array}{ccccc}
0 & -I & 0 & 0 & 0 \\
-I & P_{0, t_{i}} & 0 & \hat{A}^{T} & 0 \\
0 & 0 & U^{T} \overline{\mathrm{Q}}_{\overline{\mathrm{u}}}^{\dagger} U & U^{T} \overline{\mathrm{Q}}_{\overline{\mathrm{u}}}^{\dagger} \mathrm{S}^{T} & 0 \\
0 & \hat{A} & \mathrm{~S} \overline{\mathrm{Q}}_{\overline{\mathrm{u}}}^{\dagger} U & 0 & -I \\
0 & 0 & 0 & -I & \hat{P}_{i+1}
\end{array}\right]\left[\begin{array}{c}
\hat{\lambda}_{i} \\
\hat{x}_{i} \\
\hat{u}_{i} \\
\hat{\lambda}_{i+1} \\
\hat{x}_{i+1}
\end{array}\right]=\left[\begin{array}{c}
-\bar{x}_{i} \\
0 \\
0 \\
0 \\
0
\end{array}\right] .
$$

Here $U^{T} \overline{\mathrm{Q}}_{\overline{\mathrm{u}}}^{\dagger} U \in \mathbb{S}_{++}^{n_{\hat{u}}}$ since $\mathcal{R}(U)=\mathcal{R}\left(\mathrm{S}^{T}\right) \subseteq \mathcal{R}\left(\overline{\mathrm{Q}}_{\overline{\mathrm{u}}}\right)=$ $\mathcal{R}\left(\overline{\mathrm{Q}}_{\overline{\mathrm{u}}}^{\dagger}\right)$. The last equality follows from the properties of the Moore-Penrose pseudo-inverse [30]. Note that since $\bar{Q}_{\bar{u}}$ is block diagonal, also $\bar{Q}_{\overline{\mathrm{u}}}^{\dagger}$ is block diagonal and given by

$$
\overline{\mathrm{Q}}_{\overline{\mathrm{u}}}^{\dagger}=\operatorname{blkdiag}\left(G_{0, t_{i}+1}^{\dagger}, \ldots, G_{0, t_{i}+N_{i}}^{\dagger}\right) \text {. }
$$

Lemma 8 (Tailored reduction of control inputs): Consider a UFTOC problem (32), and assume that colrank $S<m$ holds. Then, this problem can be reduced to a UFTOC problem

$$
\begin{aligned}
\min _{\hat{x}_{i}, \hat{u}_{i}, \hat{x}_{i+1}} & \frac{1}{2}\left[\begin{array}{l}
\hat{x}_{i} \\
\hat{u}_{i}
\end{array}\right]^{T}\left[\begin{array}{cc}
\hat{Q}_{x} & 0 \\
0 & \hat{Q}_{u}
\end{array}\right]\left[\begin{array}{l}
\hat{x}_{i} \\
\hat{u}_{i}
\end{array}\right]+\frac{1}{2} \hat{x}_{i+1}^{T} \hat{P}_{i+1} \hat{x}_{i+1} \\
\text { s.t. } & \hat{x}_{i}=\bar{x}_{i} \\
& \hat{x}_{i+1}=\hat{A} \hat{x}_{i}+\hat{B} \hat{u}_{i},
\end{aligned}
$$

where $\hat{Q}_{x} \in \mathbb{S}_{+}^{n_{x}}, \hat{Q}_{u} \in \mathbb{S}_{++}^{n_{\hat{u}}}$ and $\hat{B} \in \mathbb{R}^{n_{x} \times n_{\hat{u}}}$ are given by

$$
\hat{Q}_{x} \triangleq P_{0, t_{i}}, \quad \hat{Q}_{u} \triangleq U^{T} \overline{\mathrm{Q}}_{\overline{\mathrm{u}}}^{\dagger} U, \quad \hat{B} \triangleq \mathrm{S} \overline{\mathrm{Q}}_{\overline{\mathrm{u}}}^{\dagger} U,
$$

with $n_{\hat{u}} \triangleq$ colrank $\hat{B}=$ colrank $\mathrm{S} \leq n_{x}$. Furthermore, $\hat{A}$ is defined as in (33), the columns of $U$ form an orthonormal basis of $\mathcal{R}\left(\mathrm{S}^{T}\right)$, and $\overline{\mathrm{u}}_{i}$ can be obtained as

$$
\overline{\mathrm{u}}_{i}=\overline{\mathrm{Q}}_{\overline{\mathrm{u}}}^{\dagger} U \hat{u}_{i}+\left(I-\overline{\mathrm{Q}}_{\overline{\mathrm{u}}} \overline{\mathrm{Q}}_{\overline{\mathrm{u}}}^{\dagger}\right) \hat{z}_{i} .
$$

Proof: When colrank $\mathrm{S}<m$, the KKT system (38) can be reduced to the equivalent (43). By using (46), a solution to the KKT system (43) is also a primal and dual solution to the UFTOC problem (45). (47) is then directly obtained from (40).

Since the use of the preliminary feedback in (31) results in a block diagonal $\overline{\mathrm{Q}}_{\overline{\mathrm{u}}}$ with blocks given by the $G_{0, t+1}: \mathrm{s}$, $\hat{Q}_{u}$ and $\hat{B}$ in (46) can be computed efficiently by blockwise computations once $U$ is computed. The factorizations of $G_{0, t+1}$ from the computation of $K_{0, t+1}$ can be re-used here.

For the last subproblem $i=\hat{N}$, the variable $\hat{P}_{\hat{N}+1}=Q_{x, N}$ in (27) is known. Hence, the cost-to-go function for this subproblem is computed using the Riccati factorization as

$$
J\left(\hat{x}_{\hat{N}}\right)=\frac{1}{2} \hat{x}_{\hat{N}}^{T} \hat{Q}_{x, \hat{N}} \hat{x}_{\hat{N}}, \quad \hat{Q}_{x, \hat{N}} \triangleq P_{\hat{N}} .
$$

3) Tailored Riccati-based reduction algorithm: Here, the third way of reducing the control input dimension is presented. It is similar to the second way, but a transformation $\hat{u}_{i}=T \hat{v}_{i}$ is used to avoid computing the orthonormal basis $U$.

Lemma 9: Consider a UFTOC problem as in Lemma 8. Introduce the transformation $\hat{u}_{i}=T \hat{v}_{i}$ where $T \in \mathbb{R}^{n_{\hat{u}} \times n_{x}}$ has full rank, $U T=\mathrm{S}^{T}$ and $\hat{v}_{i} \in \mathbb{R}^{n_{x}}$. Then, the primal and dual solution given by $\hat{x}_{i}^{*}, \hat{v}_{i}^{*}, \hat{x}_{i+1}^{*}, \hat{\lambda}_{i}^{*}$ and $\hat{\lambda}_{i+1}^{*}$ to 


$$
\begin{aligned}
\min _{\hat{x}_{i}, \hat{v}_{i}, \hat{x}_{i+1}} & \frac{1}{2}\left[\begin{array}{c}
\hat{x}_{i} \\
\hat{v}_{i}
\end{array}\right]^{T}\left[\begin{array}{cc}
\hat{Q}_{x} & 0 \\
0 & \hat{Q}_{v}
\end{array}\right]\left[\begin{array}{c}
\hat{x}_{i} \\
\hat{v}_{i}
\end{array}\right]+\frac{1}{2} \hat{x}_{i+1}^{T} \hat{P}_{i+1} \hat{x}_{i+1} \\
\text { s.t. } & \hat{x}_{i}=\bar{x}_{i} \\
& \hat{x}_{i+1}=\hat{A} \hat{x}_{i}+\hat{B}_{v} \hat{v}_{i}
\end{aligned}
$$

with

$$
\hat{Q}_{v}=\hat{B}_{v} \triangleq \mathrm{S} \bar{Q}_{\overline{\mathrm{u}}}^{\dagger} \mathrm{S}^{T} \in \mathbb{S}_{+}^{n_{x}},
$$

is also a solution to (45) with $\hat{u}_{i}^{*}=T \hat{v}_{i}^{*}$, and the eliminated variable $\overline{\mathrm{u}}_{i}$ in (47) can be computed from

$$
\overline{\mathrm{u}}_{i}=\overline{\mathrm{Q}}_{\overline{\mathrm{u}}}^{\dagger} \mathrm{S}^{T} \hat{v}_{i}+\left(I-\overline{\mathrm{Q}}_{\overline{\mathrm{u}}}^{\dagger} \overline{\mathrm{Q}}_{\overline{\mathrm{u}}}\right) \hat{z}_{i} .
$$

Furthermore, if $n_{\hat{u}}=n_{x}$ then $\hat{Q}_{v} \in \mathbb{S}_{++}^{n_{x}}$.

Proof: By using $\hat{Q}_{v}=\hat{B}_{v}=\mathrm{S} \overline{\mathrm{Q}}_{\overline{\mathrm{u}}}^{\dagger} \mathrm{S}^{T}=T^{T} U^{T} \overline{\mathrm{Q}}_{\overline{\mathrm{u}}}^{\dagger} U T$, the KKT system for the UFTOC problem (49) is given by

$$
\left[\begin{array}{ccccc}
0 & -I & 0 & 0 & 0 \\
-I & \hat{Q}_{x} & 0 & \hat{A}^{T} & 0 \\
0 & 0 & T^{T} U^{T} \overline{\mathbf{Q}}_{\bar{u}}^{\dagger} U T & T^{T} U^{T} \overline{\mathbf{Q}}_{\bar{u}}^{\dagger} \mathrm{S}^{T} & 0 \\
0 & \hat{A} & \mathrm{~S} \overline{\mathbf{Q}}_{\bar{u}}^{\dagger} U T & 0 & -I \\
0 & 0 & 0 & -I & \hat{P}_{i+1}
\end{array}\right]\left[\begin{array}{c}
\hat{\lambda}_{i} \\
\hat{x}_{i} \\
\hat{v}_{i} \\
\hat{\lambda}_{i+1} \\
\hat{x}_{i+1}
\end{array}\right]=\left[\begin{array}{c}
-\bar{x}_{i} \\
0 \\
0 \\
0 \\
0
\end{array}\right]
$$

Now, multiply the third block row with $\left(T T^{T}\right)^{-1} T$ from the left. This does not change the solution set of (52) since $\operatorname{rank} U^{T} \bar{Q}_{\bar{u}}^{\dagger} U T=\operatorname{rank} T^{T} U^{T} \bar{Q}_{\bar{u}}^{\dagger} U T=n_{\hat{u}}$ [30]. Furthermore, by using $\hat{u}_{i}=T \hat{v}_{i}$, it follows that a solution to (52) is also a solution to the KKT system of (45), given by (43). When $n_{\hat{u}}=n_{x}, T \in \mathbb{R}^{n_{x} \times n_{x}}$ is non-singular by definition and hence $\hat{Q}_{v} \in \mathbb{S}_{++}^{n_{x}}$. Furthermore, by inserting $\hat{u}_{i}=T \hat{v}_{i}$ into (47) gives (51), which concludes the proof.

By using Lemma 9, the reduced UFTOC problem (49) can be used instead of (45). This choice of reduced UFTOC problem has $n_{x} \geq n_{\hat{u}}=$ colrank $\mathrm{S}$ control inputs, and hence (49) might have more control inputs than (45). However, the advantage when using (49) is that $\hat{Q}_{u}$ and $\hat{B}$ can be easily computed from the definitions of $\bar{Q}_{\bar{u}}$ and $S$ without having to compute the orthonormal basis $U$. It will later be seen that also less communication is usually needed in a parallel setting.

The computational procedure for both condensing and reducing a subproblem using Lemmas 5 and 9 is summarized in Algorithm 3. This algorithm is basically a Riccati factorization, and is similar to the partitioned DP algorithm presented in [14], but here the UFTOC structure is preserved.

\section{Constructing the master problem}

In Section V-B it was shown how a subproblem in the form (27) can be condensed to either (28) or (32), depending on which condensing technique that is used. Furthermore, in Section V-C it was shown that it sometimes is possible to reduce the size of the control input in different ways to obtain a smaller UFTOC problem in either of the forms (35), (45) or (49), depending on the reduction technique. Furthermore, the last subproblem $i=\hat{N}$ can be described by the cost-togo function $J\left(\hat{x}_{\hat{N}}\right)$ according to (37) and (48), respectively. Hence, by using the definition of $\hat{x}_{i}=x_{t_{i}}$ and $J\left(\hat{x}_{i+1}\right)$, it is possible to construct a master problem with shorter prediction horizon $\hat{N}<N$ from the reduced subproblems.

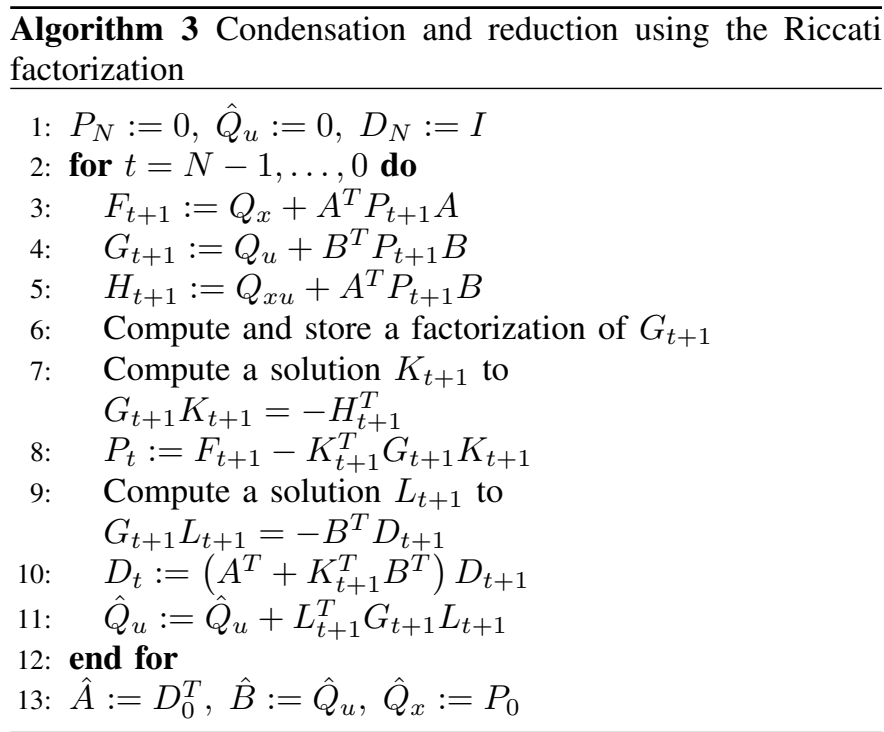

Theorem 3 (Construction of the master problem): Consider a UFTOC problem $\mathcal{P}(N)$ given in (1) where Assumption 1, and either Assumption 2 or 3, hold. Then, this problem can be reduced in parallel to the smaller master UFTOC problem

$$
\begin{aligned}
\min _{\hat{\mathrm{x}}, \hat{\mathrm{u}}} & \sum_{i=0}^{\hat{N}-1} \frac{1}{2}\left[\begin{array}{l}
\hat{x}_{i} \\
\hat{u}_{i}
\end{array}\right]^{T}\left[\begin{array}{cc}
\hat{Q}_{x} & \hat{Q}_{x u} \\
\hat{Q}_{x u}^{T} & \hat{Q}_{u}
\end{array}\right]\left[\begin{array}{l}
\hat{x}_{i} \\
\hat{u}_{i}
\end{array}\right]+\frac{1}{2} \hat{x}_{\hat{N}}^{T} \hat{Q}_{x, \hat{N}} \hat{x}_{\hat{N}} \\
\text { s.t. } & \hat{x}_{0}=\bar{x} \\
& \hat{x}_{i+1}=\hat{A} \hat{x}_{i}+\hat{B} \hat{u}_{i}, i \in \mathbb{Z}_{0, \hat{N}-1},
\end{aligned}
$$

with $\hat{N}<N, \hat{x}_{i} \in \mathbb{R}^{n_{x}}, \hat{u}_{i} \in \mathbb{R}^{n_{\hat{u}}}$, and $n_{\hat{u}} \leq n_{x}$.

Proof: Partition the UFTOC problem (1) into $\hat{N}+1$ subproblems in the form (25). Then, each subproblem can be condensed in either of the ways presented in Section V-B, and reduced as described in Section V-C to a subproblem with $n_{\hat{u}} \leq n_{x}$ control inputs. Since $J\left(\hat{x}_{\hat{N}}\right)$ is known from (48), and it follows from [34] that $J\left(\hat{x}_{i}\right)$ for all $i \in \mathbb{Z}_{0, \hat{N}-1}$ can be computed from the solution of

$$
\begin{aligned}
\min _{\hat{u}_{i}, \hat{x}_{i+1}} & \frac{1}{2}\left[\begin{array}{l}
\hat{x}_{i} \\
\hat{u}_{i}
\end{array}\right]^{T}\left[\begin{array}{cc}
\hat{Q}_{x} & \hat{Q}_{x u} \\
\hat{Q}_{x u}^{T} & \hat{Q}_{u}
\end{array}\right]\left[\begin{array}{l}
\hat{x}_{i} \\
\hat{u}_{i}
\end{array}\right]+J\left(\hat{x}_{i+1}\right) \\
\text { s.t. } & \hat{x}_{i+1}=\hat{A} \hat{x}_{i}+\hat{B} \hat{u}_{i},
\end{aligned}
$$

it follows by induction that the master problem can be defined as (53). Furthermore, the condensing and reduction of each subproblem can be performed independently of each other.

The structure of the master UFTOC problem (53) depends on which condensing and reduction technique that has been used. When the condensing technique based on the Riccati recursion is used, the condensed problem is given by (32) and hence $\hat{Q}_{x u}=0$ for such problems. Besides this, if Algorithm 3 is used then also $\hat{Q}_{u}=\hat{B} \in \mathbb{S}_{+}^{n_{x}}$ holds. Furthermore, if the original UFTOC problem (1) satisfies Assumption 2, then also the master problem satisfies Assumption 2 unless the subproblems are reduced using Algorithm 3, in which case the master UFTOC problem instead satisfies Assumption 3.

The master problem (53) can be solved using any suitable method to compute the solution $\hat{u}_{i}^{*}$ for $i \in \mathbb{Z}_{0, \hat{N}-1}$, and $\hat{x}_{i}^{*}$ 
and $\hat{\lambda}_{i}^{*}$ for $i \in \mathbb{Z}_{0, \hat{N}}$. If the Riccati recursion is used then also the cost-to-go function $\hat{P}_{i}$ at each stage $i$ is computed.

Remark 3: When Lemma 9 is used to reduce a subproblem it is possible that $\hat{Q}_{u}=\hat{Q}_{v}$ are singular. Even in such cases, there will always exist a solution to the master problem since $\hat{Q}_{u}=\hat{B}$ according to Assumption 3. However, the optimal control input will be computed as

$$
\hat{u}_{i}=\hat{K}_{i+1} \hat{x}_{i}+\hat{u}_{\mathcal{N}, i}, \quad \hat{u}_{\mathcal{N}, i} \in \mathcal{N}\left(\hat{G}_{i+1}\right),
$$

and is not unique, see for example [5], [22]. From Lemma 7 it follows that $\mathcal{N}\left(\hat{G}_{i+1}\right)=\mathcal{N}(\hat{B})$. Hence, by using the state recursion in Algorithm 2 it follows that the optimal states and dual variables $\hat{x}_{i+1}^{*}$ and $\hat{\lambda}_{i+1}^{*}$ are still unique even in this case.

Once the master problem is solved, the solution to the original UFTOC problem (1) can be obtained from the solutions to all subproblems (27) by using the definitions of $\mathrm{x}_{i}$ and $\mathrm{u}_{i}$ in (24) together with $\hat{x}_{i}=x_{t_{i}}$ and $\hat{\lambda}_{i}=\lambda_{t_{i}}$. The solutions to the subproblems can be computed in two different ways; by substituting the optimal solution to the master problem, or by re-solving the subproblems in the form in (27) using $\hat{x}_{i}^{*}$ and the (now known) cost-to-go function $J\left(\hat{x}_{i+1}\right)$. These will be described in Section V-E and V-F, respectively.

Remark 4: The solution of each subproblem can be computed independently of the other subproblems. Hence, the solution to the original UFTOC problem (1) can be computed in parallel, given the solution to the master problem (53).

\section{E. Solving the subproblems using the solution to the master problem}

Depending on which approach that has been used to condense and reduce a subproblem, the solution to the subproblem can be computed in different ways using the solution to the master problem. Here, these are presented in detail.

1) The control input dimension is reduced using Lemma 6: The optimal control input $u_{i}^{*}$ can be computed directly from $\hat{x}_{i}^{*}$ and $\hat{u}_{i}^{*}$ using (36). The optimal local variables $x_{i}^{*}$ and the optimal dual variables $\lambda_{i}^{*}$ corresponding to the local constraints can be computed from (29) using $\mathrm{u}_{i}^{*}$. For subproblems where Assumption 2 holds and that are condensed using the tailored approach presented in Lemma 5, the solution is computed analogously but where the specific structure of the condensed problem (32) is exploited. For the last subproblem $i=\hat{N}$, the solution from (37) for $\hat{x}_{\hat{N}}^{*}$ can be used.

2) Using the tailored condensation and reduction techniques: For problems that have been condensed using Lemma 5 and reduced using the approaches in Lemmas 8 or 9 , the optimal $\overline{\mathrm{u}}_{i}^{*}$ can be computed from (47) or (51) as

$$
\overline{\mathrm{u}}_{i}^{*}=\overline{\mathrm{Q}}_{\overline{\mathrm{u}}}^{\dagger} M \hat{u}_{i}^{*}+\left(I-\overline{\mathrm{Q}}_{\overline{\mathrm{u}}}^{\dagger} \overline{\mathrm{Q}}_{\overline{\mathrm{u}}}\right) \hat{z}_{i}
$$

for some $\hat{z}_{i} \in \mathbb{R}^{m}$. Here, $M$ in (56) is given by $M \triangleq U$ if Lemma 8 is used to reduce the subproblem, and by $M \triangleq \mathrm{S}^{T}$ if Lemma 9 is used. When Lemma 9 is used, and if $\hat{Q}_{u}$ is singular, then the optimal control input $\hat{u}_{i}^{*}$ for the master problem is not unique according to Remark 3. However, $\overline{\mathrm{Q}}_{\overline{\mathrm{u}}}^{\dagger} \mathrm{S}^{T} \hat{u}_{i}^{*}$ is still unique, which follows from Lemma 10:

Lemma 10: Let Assumption 3 hold and $\hat{G}_{i+1}=\hat{Q}_{u}+$ $\hat{B}^{T} \hat{P}_{i+1} \hat{B}$. Then $\mathcal{N}\left(\hat{G}_{i+1}\right) \subseteq \mathcal{N}\left(\overline{\mathrm{Q}}_{\overline{\mathrm{u}}}^{\dagger} \mathrm{S}^{T}\right)$.
Proof: If $\hat{Q}_{u} \in \mathbb{S}_{++}^{n_{\hat{u}}}$, then $\mathcal{N}\left(\hat{G}_{i+1}\right)=\{0\}$. If $\hat{Q}_{u}$ is singular, then it follows from Assumption 3 and Lemma 7 that $\mathcal{N}\left(\hat{G}_{i+1}\right)=\mathcal{N}(\hat{B})$. Now, take an arbitrary $z \in$ $\mathcal{N}\left(\hat{G}_{i+1}\right)=\mathcal{N}(\hat{B})$. From the definition of $\hat{B}=\hat{B}_{v}$ in (50) it follows that $z^{T} \hat{B} z=z^{T} \mathrm{SQ}_{\overline{\mathrm{u}}}^{\dagger} \mathrm{S}^{T} z=0$. Finally, $z^{T} \mathrm{~S} \overline{\mathrm{Q}}_{\overline{\mathrm{u}}}^{\dagger} \mathrm{S}^{T} z=0 \Longleftrightarrow \overline{\mathrm{Q}}_{\overline{\mathrm{u}}}^{\dagger} \mathrm{S}^{T} z=0$ since $\overline{\mathrm{Q}}_{\overline{\mathrm{u}}}^{\dagger} \in \mathbb{S}_{+}^{m}$.

By using the solution (56) in (31), the state recursion in Algorithm 2 and the equation for the dual variables in (34), and also noting that $x_{t_{i}}^{*}=\hat{x}_{i}^{*}$ by definition, the primal and dual solution to subproblem $i$ can be computed from

$$
\begin{aligned}
u_{t}^{*} & =K_{0, t+1} x_{t}^{*}+\bar{u}_{t}^{*}, t \in \mathbb{Z}_{t_{i}, t_{i}+N_{i}-1}, \\
x_{t+1}^{*} & =A x_{t}^{*}+B u_{t}^{*}, t \in \mathbb{Z}_{t_{i}, t_{i}+N_{i}-2}, \\
\lambda_{t}^{*} & =P_{0, t} x_{t}^{*}+D_{t}^{T} \hat{\lambda}_{i+1}^{*}, t \in \mathbb{Z}_{t_{i}+1, t_{i}+N_{i}-1} .
\end{aligned}
$$

Here, $P_{0, t}$ and $K_{0, t+1}$ were computed when the subproblem was condensed, and

$$
D_{t}=\prod_{\tau=t}^{t_{i}+N_{i}-1}\left(A+B K_{0, \tau+1}\right),
$$

is computed as in Algorithm 3. Note that the Riccati factorization in Algorithm 1 does not need to be re-computed.

Furthermore, whenever $u_{t}^{*}$ is not unique it follows from Assumption 3 that $Q_{u}=B$. Hence, from Lemma 7 it follows that $\mathcal{N}\left(G_{0, t+1}\right)=\mathcal{N}(B)$. Using this and the fact that $\hat{x}_{i}^{*}$ and $\hat{\lambda}_{i+1}^{*}$ are unique, it follows that the optimal states $x_{t}^{*}$ and dual variables $\lambda_{t}^{*}$ for $t \in \mathbb{Z}_{t_{i}, t_{i}+N_{i}}$ are still unique.

The solution to the last subproblem $i=\hat{N}$ can be computed using the state recursion in Algorithm 2 given the optimal $\hat{x}_{\hat{N}}^{*}=x_{t_{\hat{N}}}^{*}$ and the already computed Riccati factorization.

\section{F. Solving the subproblems using the cost-to-go function}

The other approach to compute the solution to a subproblem uses the cost-to-go functions $J\left(\hat{x}_{i+1}\right)$, which must be returned from the algorithm that is used to solve the master UFTOC problem in (53). The cost-to-go functions can be obtained by for example solving the master UFTOC problem using the Riccati recursion. Once the solutions $\hat{x}_{i}^{*}$ and cost-to-go functions have been obtained, the solution to each subproblem can be computed by solving (27) using the now known cost-togo function $J\left(\hat{x}_{i+1}\right)$ and initial value $\bar{x}_{i}=\hat{x}_{i}^{*}$ from the master problem. Note that for the last subproblem, the solution can be obtained from (37) since the cost-to-go function is always known for the last subproblem according to Remark 1.

One way to solve the subproblems once $\hat{x}_{i}^{*}$ and $J\left(\hat{x}_{i+1}\right)$ are known is to use the Riccati recursion. From the definition of the subproblems and the notation $\hat{P}_{i}=P_{t_{i}}$ etc., it follows that the Riccati recursion for the original UFTOC problem in the interval $t_{i} \leq t \leq t_{i+1}$ can be computed. Hence, the Riccati recursion for the original UFTOC problem can be computed in parallel from the $\hat{N}+1$ intervals $i \in \mathbb{Z}_{0, \hat{N}}$. Note that only the uniquely defined $\hat{x}_{i}^{*}$ and $J\left(\hat{x}_{i+1}\right)$ are used to compute the solution to the subproblem. Hence, the possibly non-unique $\hat{u}_{i}^{*}$ in the master problem does not need to be considered when re-solving the subproblems using the cost-to-go function. 


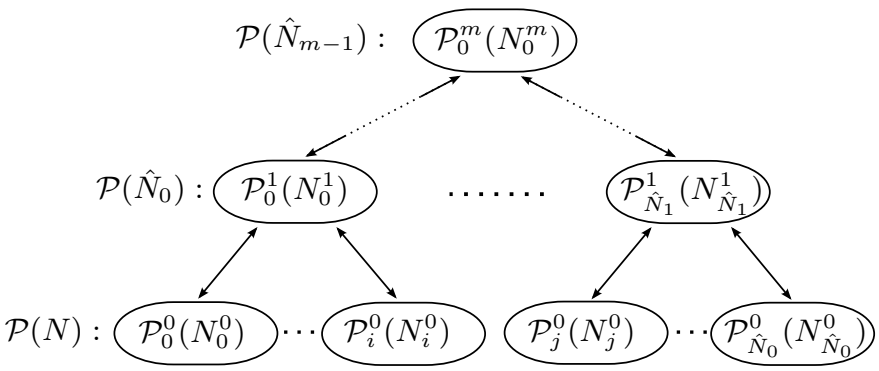

Fig. 2: The tree structure that is obtained when a UFTOC problem is reduced in $m$ steps. Each level in the tree forms a UFTOC problem that is again split into several smaller problems. $\mathcal{P}_{i}^{k}\left(N_{i}^{k}\right)$ denotes a subproblem at level $k$ in the tree in either of the forms (8) or (27) with prediction horizon $N_{i}^{k}$.

\section{Vi. Computing the Search Direction in Parallel}

In Sections IV and V, different approaches for both reducing the original UFTOC problem (1) to a master UFTOC problem, and for computing the solution to the original UFTOC problem using the solution to the master UFTOC problem were presented. Since all subproblems can be reduced independently of each other, the construction of the master UFTOC problem can be done in parallel on several computational units. Furthermore, once the solution to the master problem is computed, the subproblems can be solved independently of each other. Hence, both forming the master problem and solving the subproblems can be done in parallel. The solution to the original UFTOC problem is in principle formed by stacking the solutions to the subproblems, and hence the solution to the original UFTOC problem can be computed in parallel.

One of the key benefits with the reduction techniques presented in this paper and in [22] is that the master problem is in the same form as the original UFTOC problem, and that it satisfies the same assumptions as the UFTOC problem that is reduced. Hence, instead of solving the master UFTOC problem in Fig. 1 serially, it can itself be reduced recursively in several a priori determined steps to an even smaller UFTOC problem. This small UFTOC problem can be solved serially, and the solution can be propagated to all the intermediate UFTOC problems to finally compute the solution to the original UFTOC problem. This procedure can be described by the tree in Fig. 2, where each level $k$ in the tree is a UFTOC problem with horizon $\hat{N}_{k-1}$ and the original UFTOC problem (1) is at the bottom level. The UFTOC problem at each level is split into $\hat{N}_{k}$ subproblems which are denoted $\mathcal{P}_{i}^{k}\left(N_{i}^{k}\right)$ for $i \in \mathbb{Z}_{0, \hat{N}_{k}}$. Communication is only required between the parent and child nodes in the tree, and hence each level $k$ can be solved in parallel provided that $\hat{N}_{k}$ computational units are available.

Remark 5: Note that the sizes of the subproblems in Fig. 2 can be different, both within and between levels. Depending on for example the communication and memory layout on the hardware, different sizes of $\mathcal{P}_{i}^{k}\left(N_{i}^{k}\right)$ can be used to reduce the total computation time. This can for example be investigated by benchmarking the algorithms for the desired hardware.

\section{A. Algorithms for parallel computation of search directions}

The parallel computation of the search directions presented in this paper can be separated into two main steps:
1) Reduction step: This step consists of recursively reducing the UFTOC problems in $m$ steps upwards in the tree in Fig. 2, which is summarized in Algorithm 4.

2) Solution step: This step consists of propagating the solution to the UFTOC problems in $m$ steps downwards in the tree in Fig. 2, which is summarized in Algorithm 5.

Note that the parfor-loops in Algorithms 4 and 5 can be computed in parallel using several computational units.
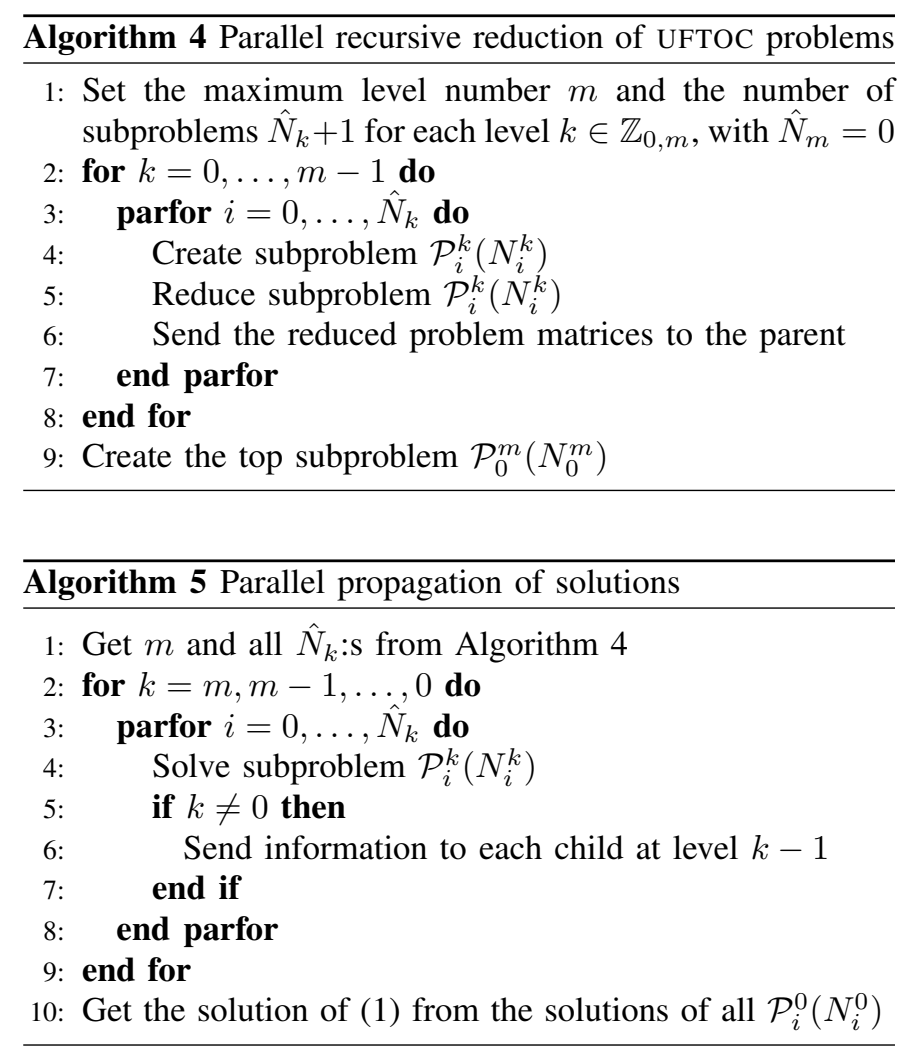

So far no assumptions on $N_{i}$ have been made. Now, assume for simplicity that $N_{i}=N_{s}$ for $i \in \mathbb{Z}_{0, \hat{N}}$, and that $N=N_{s}^{m+1}$ for some $m \in \mathbb{Z}_{++}$. Then, if there are $N_{s}^{m}$ computational units available, both the reduction and the propagation of the solution can be done in $m$ steps. Hence, the solution to the original UFTOC problem can be computed in $\mathcal{O}(\log N)$ computational complexity growth. At level $k$ in the tree in Fig. 2 only $\hat{N}_{k}$ computational units are used for reducing or solving subproblems. To use the computational resources more efficiently, e.g. standard parallel linear algebra routines can be used in the reduction and the solution of the subproblems.

The main steps in Algorithms 4 and 5 are the same for the approaches presented in Section IV and Section V. However, the details on what is sent and how the reduction and propagation of the solutions are made differ between them. These details are given below.

1) Parametric programming approach: The reduction of the subproblems at Line 5 in Algorithm 4 is done as described in Section IV-A. The matrices that are used to form the master UFTOC problem as in Theorem 1 are communicated to the parent at Line 6 in Algorithm 4. The UFTOC problem $\mathcal{P}\left(\hat{N}_{m-1}\right)$ at the top level $m$ in the tree in Fig. 2 can be solved using any method for solving UFTOC problems in the form (1). The UFTOC problems at the levels below in the tree can be 
solved as in Theorem 2 . Hence, the primal solutions $\theta_{i}^{*}$ need to be communicated to each child in the tree. For each child that is primal degenerate, also the dual solution $\hat{\lambda}_{i+1}^{*}$ needs to be communicated.

2) Parallel partial condensing and reduction approach: In the approach presented in Section $\mathrm{V}$ it is shown how to compute the solution to the UFTOC problem (1), but also how to compute the Riccati factorization in parallel. Depending on which condensing and reduction technique from Section $\mathrm{V}$ that has been used, different sets of data are used in Algorithm 4 and 5. At Line 6 in Algorithm 4 the problem matrices that are used to form the master problem (53) are sent to the parent. Note that when Lemma 5 is used to condense the subproblem then $\hat{Q}_{x u}=0$, and if it is condensed and reduced using Algorithm 3 then also $\hat{Q}_{u}=\hat{B} \in \mathbb{S}_{+}^{n_{x}}$ holds. Hence, in these cases less data need to be communicated.

The solution to the subproblems in the tree can be computed in two different ways for this approach; either by substituting the solution from the problem at the level above as described in Section V-E, or by re-solving the problem using the costto-go function $J\left(\hat{x}_{i+1}\right)$ as described in Section V-F. For the first case, the top problem $\mathcal{P}\left(\hat{N}_{m-1}\right)$ can be computed using any method for UFTOC problems in the form (1). The solution $\hat{x}_{i}^{*} \in \mathbb{R}^{n_{x}}, \hat{u}_{i}^{*} \in \mathbb{R}^{n_{\hat{u}}}$ and $\hat{\lambda}_{i+1}^{*} \in \mathbb{R}^{n_{x}}$ is communicated to the children at Line 6 in Algorithm 5. Note that for the last subproblem, only $\hat{x}_{\hat{N}}^{*} \in \mathbb{R}^{n_{x}}$ needs to be sent.

If the subproblems are re-solved using the cost-to-go function, then all subproblems, including the top problem $\mathcal{P}\left(\hat{N}_{m-1}\right)$, must be solved using an algorithm that computes the cost-to-go function for each time instance in the UFTOC problem. One example of such a method is the Riccati recursion. At Line 6 in Algorithm 5 the optimal state and costto-go function computed at level $k$ are sent to the children.

When the reduction of the subproblems is done using Algorithm 3, the condensation and reduction of the control input dimensions are done as in Lemma 5 and Lemma 9. For this case it is not certain that $\hat{Q}_{u} \in \mathbb{S}_{++}^{n_{\hat{u}}}$. For subproblems with colrank $\mathrm{S}<n_{x}$ it follows from Lemma 9 that the master UFTOC problem satisfies Assumption 3. In order to apply Algorithm 3 recursively as in Fig. 2, UFTOC problems where Assumption 3 holds must be handled. This is the motivation to why UFTOC problems that satisfy the relaxed Assumption 3 is studied in this paper.

Note that if the subproblems at the bottom level in the tree in Fig. 2 are solved using the Riccati recursion, then it follows that both the Riccati factorization and the full Riccati recursion for the original UFTOC problem (1) are computed in parallel.

\section{NUMERICAL RESULTS}

The algorithm based on the parametric approach has been implemented in MATLAB, and the algorithm based on the parallel Riccati approach using Algorithm 3 and re-computing the solution using the cost-to-go function has been implemented in both MATLAB and ANSI-C. The parallel algorithms are compared to the state-of-the-art serial Riccati recursion which is implemented in both MATLAB and ANSI-C. In all numerical experiments $N_{s}=2$ and $N / 2$ number of computational units have been used (or simulated).
In MATLAB the parallelism is simulated by executing the algorithms serially but still using the same information flow as for a parallel execution. The computation times are estimated by computing the sum of the maximum computation times for each level in the tree in Fig. 2. Hence, for the evaluations in MATLAB the communication overhead is neglected. The evaluations have been performed on an Intel Core i7-3517U CPU @ 1.9GHz running Windows 7 (version 6.1, build 7601: Service Pack 1) and MATLAB (8.0.0.783, R2012b).

The ANSI-C implementation of the parallel Riccati algorithm has been executed truly in parallel on a computer cluster consisting of nodes with 8-core Intel Xeon E5-2660 @ 2.2 $\mathrm{GHz}$ CPUs with communication over Mellanox InfiniBand FDR high-speed interconnect. The computations were performed on resources provided by the Swedish National Infrastructure for Computing (SNIC) at NSC. Hence, the evaluation of the ANSI-C implementation of the parallel Riccati algorithm includes also the communication overhead and shows the actual potential benefits using the parallel Riccati recursion compared to the serial one. The communication is based on the message-passing interface (MPI). The implementation is rudimentary, where for example no tuning of MPI parameters have been made. However, the implementation serves as a proof-of-concept that the proposed parallel Riccati algorithm actually increases performance in terms of computation time when executed truly in parallel, where the communication time is taken into consideration. The computation times for the ANSI-C implementation have been calculated using wall-clock time. Note that the scaling on the time axis of Fig. 3 in [25] is incorrect and must be multiplied by a factor $10^{3}$ to be correct.

In MATLAB, the algorithms have been evaluated by solving UFTOC problems with stable LTI systems of varying sizes and with varying prediction horizons. The computation times are averaged over 10 problems of the same dimension. The results for a UFTOC problem with $n_{x}=20$ and $n_{u}=20$ is seen in Fig. 3. From the figure it can be seen that the parallel Riccati algorithm outperforms the serial Riccati recursion for $N \gtrsim 20$. The parallel Riccati algorithm is approximately three times faster than the parametric parallel algorithm. This boost in performance is possible since the parallel Riccati recursion exploits the structure in the subproblems.

The ANSI-C implementation of the parallel Riccati algorithm has been evaluated by averaging the computation time, including the communication times, when solving 15 UFTOC problems of the same size for stable LTI systems of dimension $n_{x}=20$ and $n_{u}=20$ for different prediction horizons. In Fig. 4, the parallel algorithm is compared to a serial Riccati recursion, and it can be seen that the parallel Riccati algorithm solves a UFTOC problem with $N=512$ roughly as fast as the serial Riccati solves one with $N=45$. The parallel Riccati algorithm outperforms the serial Riccati recursion for $N \gtrsim 18$, which is similar to the results for the MATLAB implementations where the communication overhead is neglected. This speed-up can be important in for example optimal control for motion planning problems [36], [37], [38], and MHE problems where long horizons are often used [2]. 


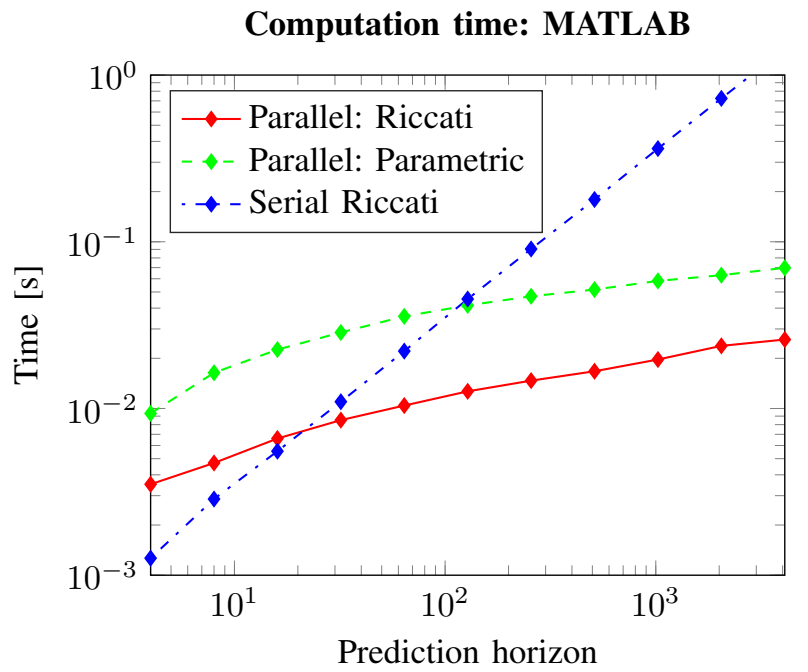

Fig. 3: Average computation times when solving UFTOC problems of order $n_{x}=20$ and $n_{u}=20$. The parallel Riccati outperforms the serial Riccati for $N \gtrsim 20$, and it is significantly faster than the parametric parallel algorithm.

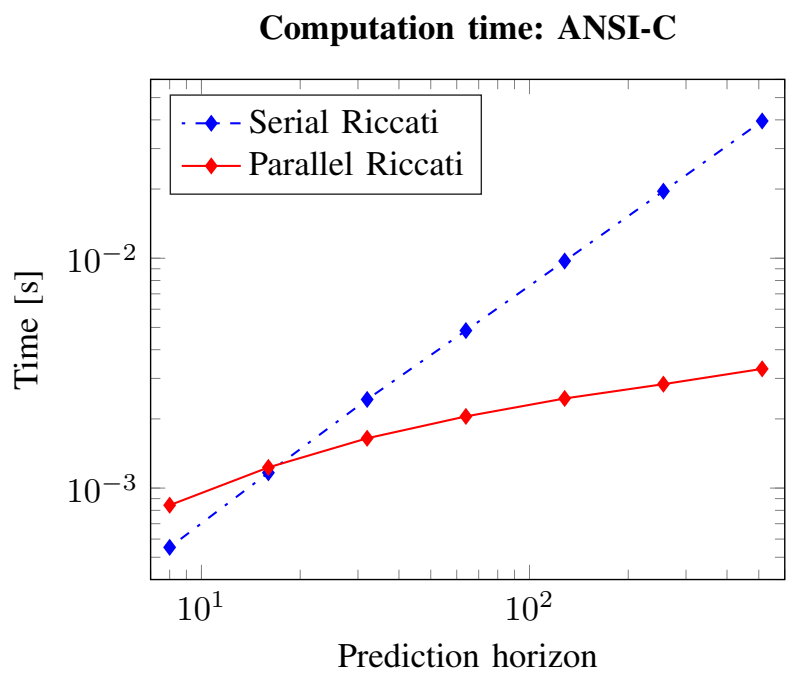

Fig. 4: Average combined computation and communication times when solving UFTOC problems of order $n_{x}=20$ and $n_{u}=20$. The parallel Riccati algorithm outperforms the serial Riccati for $N \gtrsim 18$. The parallel Riccati algorithm solves a UFTOC problem with $N=512$ roughly as fast as the serial Riccati solves one with $N=45$.

\section{CONCLUSIONS}

In this paper parallel algorithms for computing second-order search directions in MPC have been presented. The algorithms are based on a direct (non-iterative) parallel recursive approach, and the algorithms differ in the way they reduce and solve the UFTOC problems. Furthermore, it is shown how the Riccati factorization and recursion can be computed in parallel for a UFTOC problem. Numerical evaluations in MATLAB and ANSI-C are provided as proof-of-concepts for the possible performance gains, and the ANSI-C implementation is executed in parallel on a computational cluster. The numerical results indicate that significant gains in performance in terms of computation time can be achieved using the proposed algorithms.

Financial support from the Swedish Research Council (VR), the Center for Industrial Information Technology (CENIIT), and the Excellence Center at Linköping - Lund on Information Technology (ELLIIT) are hereby gratefully acknowledged.

\section{APPENDIX}

In this appendix, the definition of some problem matrices and proofs of some theorems and lemmas are presented.

A. Definition of the matrices in the UFTOC problem (27)

$$
\begin{aligned}
& \mathrm{Q}_{\mathbf{x}} \triangleq\left[\begin{array}{ccc}
Q_{x} & & \\
& \ddots & \\
& & Q_{x}
\end{array}\right], \quad \mathrm{Q}_{\mathbf{u}} \triangleq\left[\begin{array}{lll}
Q_{u} & & \\
& \ddots & \\
& & Q_{u}
\end{array}\right] \\
& \mathrm{Q}_{x \mathrm{u}} \triangleq\left[\begin{array}{llll}
Q_{x u} & 0 & \cdots & 0
\end{array}\right], \quad \mathrm{Q}_{\mathrm{xu}} \triangleq\left[\begin{array}{cccc}
0 & Q_{x u} & & \\
\vdots & & \ddots & \\
0 & & & Q_{x u}
\end{array}\right]
\end{aligned}
$$

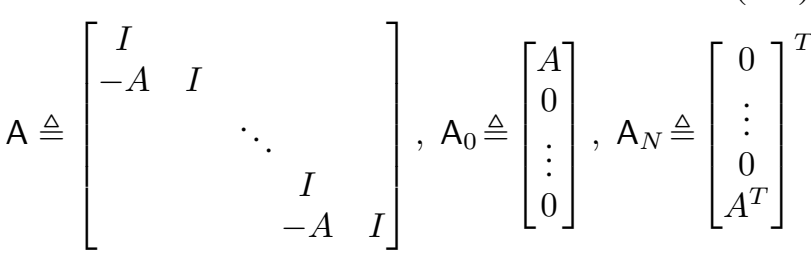

$$
\begin{aligned}
& \mathrm{B} \triangleq\left[\begin{array}{cccc}
B & & & 0 \\
& \ddots & & \vdots \\
& & B & 0
\end{array}\right], \quad \mathrm{B}_{N} \triangleq\left[\begin{array}{llll}
0 & \ldots & 0 & B
\end{array}\right] .
\end{aligned}
$$

\section{B. Definition of the matrices used in Lemma 4}

$$
\left[\begin{array}{cc}
\tilde{Q}_{x} & \tilde{Q}_{x \mathrm{u}} \\
\tilde{Q}_{x \mathrm{u}}^{T} & \tilde{Q}_{\mathrm{u}}
\end{array}\right]=\left[\begin{array}{cc}
I & 0 \\
W & Y \\
0 & I
\end{array}\right]^{T}\left[\begin{array}{ccc}
Q_{x} & 0 & \mathrm{Q}_{x \mathrm{u}} \\
0 & \mathrm{Q}_{\mathrm{x}} & \mathrm{Q}_{\mathrm{xu}} \\
\mathrm{Q}_{x \mathrm{u}}^{T} & \mathrm{Q}_{\mathrm{xu}}^{T} & \mathrm{Q}_{\mathrm{u}}
\end{array}\right]\left[\begin{array}{cc}
I & 0 \\
W & Y \\
0 & I
\end{array}\right]
$$

$\tilde{A} \triangleq \mathrm{A}_{N} W, \quad \tilde{\mathrm{B}} \triangleq \mathrm{B}_{N}+\mathrm{A}_{N} Y, \quad W \triangleq \mathrm{A}^{-1} \mathrm{~A}_{0}, \quad Y \triangleq \mathrm{A}^{-1} \mathrm{~B}$.

\section{Definition of the matrices used in Lemma 6}

$\left[\begin{array}{cc}\hat{Q}_{x} & \hat{Q}_{x u} \\ \hat{Q}_{x u}^{T} & \hat{Q}_{u}\end{array}\right] \triangleq\left[\begin{array}{cc}\tilde{Q}_{x} & \tilde{Q}_{x u} U \\ U^{T} \tilde{Q}_{x u}^{T} & U^{T} \tilde{Q}_{u} U\end{array}\right]-\left[\begin{array}{c}\tilde{Q}_{x u} V \\ U^{T} \tilde{Q}_{\mathrm{u}} V\end{array}\right] M\left[\begin{array}{c}\tilde{Q}_{x u} V \\ U^{T} \tilde{Q}_{\mathrm{u}} V\end{array}\right]^{T}$

$M \triangleq\left(V^{T} \tilde{Q}_{\mathrm{u}} V\right)^{-1}, \quad \hat{A} \triangleq \tilde{A}, \quad \hat{B} \triangleq \tilde{\mathrm{B}} U$.

The columns of $U$ form an orthonormal basis for $\mathcal{R}\left(\tilde{\mathrm{B}}^{T}\right)$ and the columns of $V$ form an orthonormal basis for $\mathcal{N}(\tilde{B})$. 


\section{Proof of Lemma 2}

Lemma 2 will be proved in three main steps; re-write (8) into an equivalent $\mathrm{mp}-\mathrm{QP}$ problem, relate the value functions between the equivalent problems, and show that $\hat{Q}_{u} \succ 0$ holds due to the structure of the introduced parametrization.

Proof: Consider the problem (8), and omit the subindex $i$ for brevity. Let $\Pi$ be a permutation matrix such that

$$
\Pi X=\left[\begin{array}{l}
\mathrm{x} \\
\mathrm{u}
\end{array}\right], \quad \Pi \mathrm{H} \Pi^{T}=\left[\begin{array}{cc}
\mathrm{H}_{x} & \mathrm{H}_{x u} \\
\mathrm{H}_{x u}^{T} & \mathrm{H}_{u}
\end{array}\right],
$$

where the elements in $\mathrm{x}$ and $\mathrm{u}$ are the vectors $x_{t}$ and $u_{t}$, respectively, and $\mathrm{H}_{u}=\operatorname{blkdiag}\left(Q_{u}, \ldots, Q_{u}\right) \succ 0$ by definition. Now, introduce the change of variables

$$
\left[\begin{array}{l}
\mathrm{x} \\
\mathrm{u}
\end{array}\right]=\underbrace{\left[\begin{array}{cc}
I & 0 \\
-\mathrm{H}_{u}^{-1} \mathrm{H}_{x u}^{T} & I
\end{array}\right]}_{\triangleq T}\left[\begin{array}{c}
\overline{\mathrm{x}} \\
\overline{\mathrm{u}}
\end{array}\right] \Longleftrightarrow\left[\begin{array}{l}
\overline{\mathrm{x}} \\
\overline{\mathrm{u}}
\end{array}\right]=\underbrace{\left[\begin{array}{cc}
I & 0 \\
\mathrm{H}_{u}^{-1} \mathrm{H}_{x u}^{T} & I
\end{array}\right]}_{=T^{-1}}\left[\begin{array}{c}
\mathrm{x} \\
\mathrm{u}
\end{array}\right] .
$$

Then the problem (8) can equivalently be formulated

$$
\begin{aligned}
\min _{\overline{\mathrm{x}}, \overline{\mathrm{u}}} & {\left[\begin{array}{l}
\overline{\mathrm{x}} \\
\overline{\mathrm{u}}
\end{array}\right]^{T}\left[\begin{array}{cc}
\mathrm{H} / \mathrm{H}_{u} & 0 \\
0 & \mathrm{H}_{u}
\end{array}\right]\left[\begin{array}{l}
\overline{\mathrm{x}} \\
\overline{\mathrm{u}}
\end{array}\right] } \\
\text { s.t. } & \mathrm{A} \Pi^{T} T\left[\begin{array}{l}
\overline{\mathrm{x}} \\
\overline{\mathrm{u}}
\end{array}\right]=\mathrm{G} \theta,
\end{aligned}
$$

where $\mathrm{H} / \mathrm{H}_{u} \succeq 0, \mathrm{H}_{u} \succ 0$, and the cross-terms are zero [39]. The unique primal parametric solution to (64) is given by

$$
\left[\begin{array}{l}
\overline{\mathbf{x}}^{*}(\hat{x}, \hat{u}) \\
\overline{\mathbf{u}}^{*}(\hat{x}, \hat{u})
\end{array}\right] \triangleq\left[\begin{array}{l}
\overline{\mathbf{x}}^{*}(\theta) \\
\overline{\mathbf{u}}^{*}(\theta)
\end{array}\right]=\left[\begin{array}{cc}
\Omega_{x} & \Omega_{u} \\
\Gamma_{x} & \Gamma_{u}
\end{array}\right]\left[\begin{array}{l}
\hat{x} \\
\hat{u}
\end{array}\right]
$$

where $\theta=\left[\begin{array}{ll}\hat{x}^{T} & \hat{u}^{T}\end{array}\right]^{T}$ is used, [32]. Now, by using the definition of $V(\theta)$ in (15), the permutation matrix $\Pi$, and the transformation $T$ in (63), it follows that

$$
\begin{aligned}
\hat{V}(\hat{x}, \hat{u}) \triangleq \hat{V}(\theta) \stackrel{(15)}{=} \frac{1}{2}\left[\begin{array}{l}
\mathrm{x}^{*}(\theta) \\
\mathbf{u}^{*}(\theta)
\end{array}\right]^{T}\left[\begin{array}{cc}
\mathrm{H}_{x} & \mathrm{H}_{x u} \\
\mathrm{H}_{x u}^{T} & \mathrm{H}_{u}
\end{array}\right]\left[\begin{array}{l}
\mathrm{x}^{*}(\theta) \\
\mathrm{u}^{*}(\theta)
\end{array}\right] \stackrel{(63)}{=} \\
\frac{1}{2}\left[\begin{array}{l}
\overline{\mathrm{x}}^{*}(\theta) \\
\overline{\mathrm{u}}^{*}(\theta)
\end{array}\right]^{T}\left[\begin{array}{cc}
\mathrm{H} / \mathrm{H}_{u} & 0 \\
0 & \mathrm{H}_{u}
\end{array}\right]\left[\begin{array}{l}
\overline{\mathrm{x}}^{*}(\theta) \\
\overline{\mathrm{u}}^{*}(\theta)
\end{array}\right] .
\end{aligned}
$$

By substituting the parametric solution (65) into (66) and expanding all expressions, the Hessian of $\hat{V}(\hat{x}, \hat{u})$ with respect to $\hat{u}$ can be identified as

$$
\nabla_{\hat{u}}^{2} \hat{V}(\hat{x}, \hat{u})=\hat{Q}_{u}=\Gamma_{u}^{T} \mathrm{H}_{u} \Gamma_{u}+\Omega_{u}^{T}\left(\mathrm{H} / \mathrm{H}_{u}\right) \Omega_{u} .
$$

Hence, $\hat{Q}_{u} \succ 0$ if $\Gamma_{u}^{T} \mathrm{H}_{u} \Gamma_{u} \succ 0$ since $\Omega_{u}^{T}\left(\mathrm{H} / \mathrm{H}_{u}\right) \Omega_{u} \succeq 0$ by definition. The final step is to show that $\Gamma_{u}^{T} \mathrm{H}_{u} \Gamma_{u} \succ 0$, which is satisfied if $\mathcal{N}\left(\Gamma_{u}\right)=\{0\}$ since $\mathrm{H}_{u} \succ 0$ by definition [30]. Assume, for contradiction, that there exists a $z \in \mathcal{N}\left(\Gamma_{u}\right) \backslash 0$. Let $\hat{u}$ and $\hat{x}$ be arbitrary parameters, and define the parameter $\hat{z} \triangleq \hat{u}+z$. Then,

$\overline{\mathrm{u}}^{*}(\hat{x}, \hat{z})=\Gamma_{x} \hat{x}+\Gamma_{u} \hat{u}+\Gamma_{u} z \stackrel{z \in \mathcal{N}\left(\Gamma_{u}\right)}{=} \Gamma_{x} \hat{x}+\Gamma_{u} \hat{u}=\overline{\mathbf{u}}^{*}(\hat{x}, \hat{u})$

holds. By expressing the terminal constraint in (64) equivalently as in (9) and exploiting that $\overline{\mathrm{u}}^{*}(\theta)$ satisfies the equality constraints (specifically the terminal constraint) in the mp-QP problem (64), it follows from (68) that

$$
\begin{aligned}
& \hat{A} \hat{x}+\hat{B} \hat{u}=\overline{\mathrm{A}} \hat{x}+\overline{\mathrm{S}} \bar{u}^{*}(\hat{x}, \hat{u}) \stackrel{(68)}{=} \overline{\mathrm{A}} \hat{x}+\overline{\mathrm{S}} \bar{u}^{*}(\hat{x}, \hat{z})= \\
& \hat{A} \hat{x}+\hat{B} \hat{z} \Longrightarrow \hat{B} \hat{u}=\hat{B} \hat{z} .
\end{aligned}
$$

By inserting the definition of $\hat{z}$ into the last equation in (69), it follows that

$$
\hat{B} \hat{u}=\hat{B} \hat{u}+\hat{B} z \Longleftrightarrow \hat{B} z=0 \Longleftrightarrow z=0,
$$

where the last equivalence follows from that $\hat{B}$ has full column rank from the definition of $\hat{B}$ in (11). However, $z=0$ contradicts the assumption of the existence of a $z \in \mathcal{N}\left(\Gamma_{u}\right) \backslash 0$. Hence, it follows that $\mathcal{N}\left(\Gamma_{u}\right)=\{0\} \Longleftrightarrow \Gamma_{u}^{T} \mathrm{H}_{u} \Gamma_{u} \succ$ $0 \Longrightarrow \hat{Q}_{u} \succ 0$, which concludes the proof.

\section{E. Proof of Theorem 2}

The KKT system for (1) consists of the equations

$$
\begin{aligned}
& Q_{x} x_{t}+Q_{x u} u_{t}-\lambda_{t}+A^{T} \lambda_{t+1}=0, t \in \mathbb{Z}_{0, N-1}, \\
& Q_{x u}^{T} x_{t}+Q_{u} u_{t}+B^{T} \lambda_{t+1}=0, t \in \mathbb{Z}_{0, N-1}, \\
& Q_{x, N} x_{N}-\lambda_{N}=0, \\
& x_{0}=\bar{x}_{0}, \quad x_{t+1}=A x_{t}+B u_{t}, t \in \mathbb{Z}_{0, N-1} .
\end{aligned}
$$

The KKT system for the extended problem (6) is given by

$$
\begin{aligned}
\mathrm{H} X_{i}+\mathrm{A}^{T} \Lambda_{i} & =0, i \in \mathbb{Z}_{0, \hat{N}}, \\
\mathrm{~A} X_{i}-\mathrm{G}\left[\begin{array}{c}
\hat{x}_{i} \\
\hat{u}_{i}
\end{array}\right] & =0, i \in \mathbb{Z}_{0, \hat{N}-1}, \\
\mathrm{~A}_{\hat{N}} X_{\hat{N}}-\mathrm{G}_{\hat{N}} \hat{x}_{\hat{N}}=0, & \\
-\hat{x}_{0}+\bar{x}=0, \quad-\hat{\lambda}_{\hat{N}}+\lambda_{0, \hat{N}} & =0, \\
-\hat{x}_{i+1}+\hat{A} \hat{x}_{i}+\hat{B} \hat{u}_{i} & =0, i \in \mathbb{Z}_{0, \hat{N}-1}, \\
-\hat{\lambda}_{i}+\lambda_{0, i}+\hat{A}^{T}\left(\lambda_{t c, i}+\hat{\lambda}_{i+1}\right) & =0, i \in \mathbb{Z}_{0, \hat{N}-1}, \\
\hat{B}^{T}\left(\lambda_{t c, i}+\hat{\lambda}_{i+1}\right) & =0, i \in \mathbb{Z}_{0, \hat{N}-1},
\end{aligned}
$$

where $X_{i}, \Lambda_{i}$ and $\hat{\lambda}_{i}$ are defined as in (5c), (13) and (18).

Proof: From the last block rows in (72a) and (72b), and (72e) it follows that for $i \in \mathbb{Z}_{0, \hat{N}-1}$ it holds that

$$
x_{N_{i}, i}^{*}=x_{0, i+1}^{*}, \quad \lambda_{N_{i}, i}^{*}=-\lambda_{t c, i}^{*} .
$$

For subproblems where LICQ holds it follows that rank $\tilde{B}=$ rank $\hat{B}=n_{x}$, and hence it follows from (72f) and (72g) that

$$
\lambda_{t c, i}^{*}=-\hat{\lambda}_{i+1}^{*}, \quad \hat{\lambda}_{i}^{*}=\lambda_{0, i}^{*} .
$$

For subproblems where LICQ is violated, the dual solution is computed as in (22) with $\xi_{t c, i}^{*}$ defined as in (23). Using this choice of dual variables in the subproblem results in $\lambda_{t c, i}^{*}=$ $\hat{\lambda}_{i+1}^{*}$ due to the definition of $Z$ and $\xi_{t c, i}^{*}$, and hence (74) holds also for the subproblems where LICQ is violated.

The solutions $X_{i}^{*}$ for $i \in \mathbb{Z}_{0, \hat{N}}$ to the subproblems satisfy the dynamics constraints (72b). Furthermore, due to (73)

$$
x_{0, i+1}^{*}=A x_{N_{i}-1, i}^{*}+B u_{N_{i}-1, i}^{*},
$$

holds for all $i \in \mathbb{Z}_{0, \hat{N}-1}$. Hence, it follows that the primal solution (21) satisfies the dynamics constraints (71d).

Furthermore, $\Lambda_{i}^{*}$ satisfies (72a) for $i \in \mathbb{Z}_{0, \hat{N}}$ and together with (73) and (74) it follows that the solution (21) satisfies (71a)-(71c), and hence (21) is a solution to (1), [23]. 


\section{F. Proof of Lemma 3}

Proof: From the definition of $\mathrm{A}$ in $(5 \mathrm{~b})$ and $\mathcal{N}\left(\mathrm{A}^{T}\right)=$ $\left\{\lambda_{i}^{\mathcal{N}} \in \mathbb{R}^{m} \mid \mathrm{A}^{T} \lambda_{i}^{\mathcal{N}}=0\right\}$ it follows that

$$
\begin{aligned}
-\lambda_{t, i}^{\mathcal{N}}+A^{T} \lambda_{t+1, i}^{\mathcal{N}} & =0, t \in \mathbb{Z}_{0, N_{i}-1}, \\
B^{T} \lambda_{t+1, i}^{\mathcal{N}} & =0, t \in \mathbb{Z}_{0, N_{i}-1}^{\mathcal{N}}, \\
\lambda_{N_{i}, i}^{\mathcal{N}} & =-\lambda_{t c, i}^{\mathcal{N}},
\end{aligned}
$$

must hold. Equations (76a) and (76c) can be combined into

$$
\lambda_{i}^{\mathcal{N}}=\left[\begin{array}{lll}
-\tilde{\mathrm{A}} & -\tilde{\mathrm{D}} & I
\end{array}\right]^{T} \lambda_{t c, i}^{\mathcal{N}}
$$

where $\tilde{D}$ and $\tilde{A}$ are defined as in (10a). By combining (76a) and (76b) and using (76c) it follows that $-\tilde{\mathrm{B}}^{T} \lambda_{t c, i}^{\mathcal{N}}=0$ holds. Here $\tilde{\mathrm{B}}$ is defined in (10b). Hence $\lambda_{t c, i}^{\mathcal{N}} \in \mathcal{N}\left(\tilde{\mathrm{B}}^{T}\right)$ must hold.

\section{G. Proof of Lemma 4}

Proof: The KKT system for (27) is given by

$\left[\begin{array}{ccc|cc|cc}0 & -I & 0 & 0 & 0 & 0 & 0 \\ -I & Q_{x} & \mathrm{Q}_{x \mathrm{u}} & 0 & \mathrm{~A}_{0}^{T} & 0 & 0 \\ 0 & \mathrm{Q}_{x \mathrm{u}}^{T} & \mathrm{Q}_{\mathrm{u}} & \mathrm{Q}_{\mathrm{xu}}^{T} & \mathrm{~B}^{T} & \mathrm{~B}_{N}^{T} & 0 \\ \hline 0 & 0 & \mathrm{Q}_{\mathrm{xu}} & \mathrm{Q}_{\mathrm{x}} & -\mathrm{A}^{T} & \mathrm{~A}_{N}^{T} & 0 \\ 0 & \mathrm{~A}_{0} & \mathrm{~B} & -\mathrm{A} & 0 & 0 & 0 \\ \hline 0 & 0 & \mathrm{~B}_{N} & \mathrm{~A}_{N} & 0 & 0 & -I \\ 0 & 0 & 0 & 0 & 0 & -I & \hat{P}_{i+1}\end{array}\right]\left[\begin{array}{c}\hat{\lambda}_{i} \\ \hat{x}_{i} \\ \mathrm{u}_{i} \\ \hline \mathrm{x}_{i} \\ \lambda_{i} \\ \hat{\lambda}_{i+1} \\ \hat{x}_{i+1}\end{array}\right]=\left[\begin{array}{c}-\bar{x} \\ 0 \\ 0 \\ \hline 0 \\ 0 \\ \hline 0 \\ 0\end{array}\right]$

Since $A$ is invertible, the second block matrix on the diagonal is also invertible and hence $x_{i}$ and $\lambda_{i}$ can be computed as

$$
\left[\begin{array}{c}
\mathrm{x}_{i} \\
\lambda_{i}
\end{array}\right]=-\left[\begin{array}{cc}
\mathrm{Q}_{\mathrm{x}} & -\mathrm{A}^{T} \\
-\mathrm{A} & 0
\end{array}\right]^{-1}\left(\left[\begin{array}{cc}
0 & \mathrm{Q}_{\mathrm{xu}} \\
\mathrm{A}_{0} & \mathrm{~B}
\end{array}\right]\left[\begin{array}{c}
\hat{x}_{i} \\
\mathrm{u}_{i}
\end{array}\right]+\left[\begin{array}{c}
\mathrm{A}_{N}^{T} \\
0
\end{array}\right] \hat{\lambda}_{i+1}\right) \text {. }
$$

Then (29) follows directly from (79). Furthermore, using (79) to eliminate $\mathrm{x}_{i}$ and $\lambda_{i}$ from the KKT system (78) gives

$$
\left[\begin{array}{ccccc}
0 & -I & 0 & 0 & 0 \\
-I & \tilde{Q}_{x} & \tilde{Q}_{x \mathrm{u}} & \tilde{A}^{T} & 0 \\
0 & \tilde{Q}_{x \mathrm{u}}^{T} & \tilde{Q}_{\mathrm{u}} & \tilde{\mathrm{B}}^{T} & 0 \\
0 & \tilde{A} & \tilde{\mathrm{B}} & 0 & -I \\
0 & 0 & 0 & -I & \hat{P}_{i+1}
\end{array}\right]\left[\begin{array}{c}
\hat{\lambda}_{i} \\
\hat{x}_{i} \\
\mathrm{u}_{i} \\
\hat{\lambda}_{i+1} \\
\hat{x}_{i+1}
\end{array}\right]=\left[\begin{array}{c}
-\bar{x} \\
0 \\
0 \\
0 \\
0
\end{array}\right]
$$

where the matrices are defined as in (60). The cost matrix in (60) is positive semi-definite by construction. Furthermore, since the KKT system (78) has a unique solution due to that Assumptions 1, 2 and LICQ hold, the reduced Hessian of the corresponding UFTOC problem is positive definite [29], [23]. From this it follows that $\tilde{Q}_{\mathrm{u}} \succ 0$ holds. The reduced system of equations (80) can be recognized as the KKT optimality conditions for the QP problem (28).

\section{H. Proof of Lemma 5}

Proof: The KKT system for the UFTOC problem (27) is

$$
\begin{gathered}
-x_{t_{i}}+\bar{x}_{i}=0, \quad-\hat{\lambda}_{i+1}+\hat{P}_{i+1} \hat{x}_{i+1}=0 \\
{\left[\begin{array}{ccccc}
-I & Q_{x} & Q_{x u} & A^{T} & 0 \\
0 & Q_{x u}^{T} & Q_{u} & B^{T} & 0 \\
0 & A & B & 0 & -I
\end{array}\right]\left[\begin{array}{c}
\lambda_{t} \\
x_{t} \\
u_{t} \\
\lambda_{t+1} \\
x_{t+1}
\end{array}\right]=\left[\begin{array}{l}
0 \\
0 \\
0
\end{array}\right], t \in \mathbb{Z}_{t_{i}, t_{i}+N_{i}-1}}
\end{gathered}
$$

Let $P_{0, t}$ and $K_{0, t}$ be obtained from the Riccati factorization using the preliminary choice $\hat{P}_{i+1}=0$, giving $P_{0, t_{i}+N_{i}}=0$. Assume that $\lambda_{t}$ for some $t \in \mathbb{Z}_{t_{i}, t_{i}+N_{i}}$ can be computed from

$$
\lambda_{t}=P_{0, t} x_{t}+D_{t}^{T} \hat{\lambda}_{i+1},
$$

when $\hat{P}_{i+1} \neq 0$. Clearly this holds at the end point $\lambda_{t_{i}+N_{i}}=$ $\hat{\lambda}_{i+1}$ if $D_{t_{i}+N_{i}} \triangleq I$. Now, by using (82) for $t+1$ together with $x_{t+1}=A x_{t}+B u_{t}$ to eliminate $\lambda_{t+1}$ from (81), exploiting the relation $H_{0, t+1}=-K_{0, t+1}^{T} G_{0, t+1}$ by the definition of $K_{0, t+1}$, and using (31), it is possible to re-write (72) into

$\left[\begin{array}{ccccc}-I & P_{0, t} & 0 & 0 & D_{t}^{T} \\ 0 & 0 & G_{0, t+1} & 0 & B^{T} D_{t+1}^{T} \\ 0 & \left(A+B K_{0, t+1}\right) & B & -I & 0\end{array}\right]\left[\begin{array}{c}\lambda_{t} \\ x_{t} \\ \bar{u}_{t} \\ x_{t+1} \\ \hat{\lambda}_{i+1}\end{array}\right]=\left[\begin{array}{l}0 \\ 0 \\ 0\end{array}\right]$

where $D_{t} \triangleq D_{t+1}\left(A+B K_{0, t+1}\right)$. Hence (82) holds also for time $t$. Since $t$ is chosen arbitrary and $D_{t_{i}+N_{i}}=I$ it follows from induction that (34b) holds for all $t \in \mathbb{Z}_{t_{i}, t_{i}+N_{i}}$.

By using the state recursion $x_{t+1}=\left(A+B K_{0, t+1}\right) x_{t}+$ $B \bar{u}_{t}$, the states $x_{t}$ for $t \in \mathbb{Z}_{t_{i}+1, t_{i}+N_{i}-1}$ can be eliminated from the system of equations (83) to obtain the KKT system of the UFTOC problem (32). This concludes the proof.

\section{Proof of Lemma 6}

Proof: The KKT system for (28) is

$$
\left[\begin{array}{ccccc}
0 & -I & 0 & 0 & 0 \\
-I & \tilde{Q}_{x} & \tilde{Q}_{x \mathrm{u}} & \tilde{A}^{T} & 0 \\
0 & \tilde{Q}_{x \mathrm{u}}^{T} & \tilde{Q}_{\mathrm{u}} & \tilde{\mathrm{B}}^{T} & 0 \\
0 & \tilde{A} & \tilde{\mathrm{B}} & 0 & -I \\
0 & 0 & 0 & -I & \hat{P}_{i+1}
\end{array}\right]\left[\begin{array}{c}
\hat{\lambda}_{i} \\
\hat{x}_{i} \\
\mathrm{u}_{i} \\
\hat{\lambda}_{i+1} \\
\hat{x}_{i+1}
\end{array}\right]=\left[\begin{array}{c}
-\bar{x} \\
0 \\
0 \\
0 \\
0
\end{array}\right]
$$

Since colrank $\tilde{\mathrm{B}}=n_{\hat{u}}<m$ holds, it is possible to find a unitary matrix $[U V]$ where the columns of $U \in \mathbb{R}^{m \times n_{\hat{u}}}$ form an orthonormal basis for $\mathcal{R}\left(\tilde{\mathrm{B}}^{T}\right)$ and the columns of $V \in$ $\mathbb{R}^{m \times\left(m-n_{\hat{u}}\right)}$ form an orthonormal basis of $\mathcal{N}(\tilde{\mathrm{B}})$. Hence, the vector $\mathrm{u}_{i}$ can be decomposed as

$$
\mathrm{u}_{i}=U \hat{u}_{i}+V \hat{z}_{i}=\left[\begin{array}{ll}
U & V
\end{array}\right]\left[\begin{array}{l}
\hat{u}_{i} \\
\hat{z}_{i}
\end{array}\right],
$$

where $\hat{u}_{i} \in \mathbb{R}^{n_{\hat{u}}}$ and $\hat{z}_{i} \in \mathbb{R}^{m-n_{\hat{u}}}$ [30]. By using (85) and multiplying the third row in (84) from the left with the matrix $[U V]^{T}$ gives the equivalent system of equations

$$
\left[\begin{array}{cccccc}
0 & -I & 0 & 0 & 0 & 0 \\
-I & \tilde{Q}_{x} & \tilde{Q}_{x \mathrm{u}} U & \tilde{Q}_{x \mathrm{u}} V & \tilde{A}^{T} & 0 \\
0 & U^{T} \tilde{Q}_{x \mathrm{u}}^{T} & U^{T} \tilde{Q}_{\mathrm{u}} U & U^{T} \tilde{Q}_{\mathrm{u}} V & U^{T} \tilde{\mathrm{B}}^{T} & 0 \\
0 & V^{T} \tilde{Q}_{x \mathrm{u}}^{T} & V^{T} \tilde{Q}_{\mathrm{u}} U & V^{T} \tilde{Q}_{\mathrm{u}} V & 0 & 0 \\
0 & \tilde{A}_{0} & \tilde{\mathrm{B}} U & 0 & 0 & -I \\
0 & 0 & 0 & 0 & -I & \hat{P}_{i+1}
\end{array}\right]\left[\begin{array}{c}
\hat{\lambda}_{i} \\
\hat{x}_{i} \\
\hat{u}_{i} \\
\hat{z}_{i} \\
\hat{\lambda}_{i+1} \\
\hat{x}_{i+1}
\end{array}\right]=\left[\begin{array}{c}
-\bar{x} \\
0 \\
0 \\
0 \\
0 \\
0
\end{array}\right]
$$

Here the relation $\tilde{\mathrm{B}} V=0$ has been used. Since $\tilde{Q}_{\mathrm{u}} \succ 0$ and $V$ has full rank, $V^{T} \tilde{Q}_{\mathrm{u}} V \succ 0$ and $\hat{z}_{i}$ can be eliminated from (86) using the Schur complement. Then, the resulting system of equations is the KKT system for the reduced UFTOC problem (28), and $\hat{z}_{i}$ can be computed as

$$
\hat{z}_{i}=-\left(V^{T} \tilde{Q}_{\mathrm{u}} V\right)^{-1}\left(V^{T} \tilde{Q}_{x \mathrm{u}}^{T} \hat{x}_{i}+V^{T} \tilde{Q}_{\mathrm{u}} U \hat{u}_{i}\right) .
$$


Positive-semidefiniteness of (61a) follows from the convexity of the objective function in (28) and the properties of the Schur complement. Furthermore, $\hat{Q}_{u} \succ 0$ follows from $\tilde{Q}_{\mathrm{u}} \succ 0$, and (36) follows from (85) and (87).

\section{REFERENCES}

[1] H. Jonson, "A Newton method for solving non-linear optimal control problems with general constraints," Ph.D. dissertation, Linköping University, 1983.

[2] C. V. Rao, S. J. Wright, and J. B. Rawlings, "Application of interior-point methods to model predictive control," Journal of Optimization Theory and Applications, vol. 99, no. 3, pp. 723-757, 1998.

[3] L. Vandenberghe, S. Boyd, and M. Nouralishahi, "Robust linear programming and optimal control," Department of Electrical Engineering, University of California Los Angeles, Tech. Rep., 2002.

[4] D. Axehill, A. Hansson, and L. Vandenberghe, "Relaxations applicable to mixed integer predictive control - comparisons and efficient computations," in Proceedings of the 46th IEEE Conference on Decision and Control, New Orleans, LA, USA, Dec. 2007, pp. 4103-4109.

[5] D. Axehill, "Integer quadratic programming for control and communication," Ph.D. dissertation, Linköping University, 2008.

[6] D. Axehill and A. Hansson, "A dual gradient projection quadratic programming algorithm tailored for model predictive control," in Proceedings of the 47th IEEE Conference on Decision and Control, Cancun, Mexico, Dec. 2008, pp. 3057-3064.

[7] M. Diehl, H. J. Ferreau, and N. Haverbeke, Efficient Numerical Methods for Nonlinear MPC and Moving Horizon Estimation. Springer-Verlag, 2009.

[8] C. Kirches, H. G. Bock, J. P. Schlöder, and S. Sager, "A factorization with update procedures for a KKT matrix arising in direct optimal control," Mathematical Programming Computation, vol. 3, no. 4, pp. 319-348, 2011.

[9] I. Nielsen, D. Ankelhed, and D. Axehill, "Low-rank modifications of Riccati factorizations with applications to model predictive control," in Proceedings of the 52nd IEEE Conference on Decision and Control, Florence, Italy, Dec. 2013, pp. 3684-3690.

[10] T. S. Chang and P. B. Luh, "On the decomposition and coordination of large scale dynamic control problems," in Proceedings of the 24th IEEE Conference on Decision and Control, Fort Lauderdale, FL, USA, Dec. 1985, pp. 1484-1485.

[11] T. S. Chang, X. X. Jin, P. B. Luh, and X. Miao, "Large-scale convex optimal control problems: time decomposition, incentive coordination, and parallel algorithm," IEEE Transactions on Automatic Control, vol. 35, no. 1 , pp. 108-114, 1990.

[12] S. C. Chang, T. S. Chang, and P. B. Luh, "A hierarchical decomposition for large-scale optimal control problems with parallel processing structure," Automatica, vol. 25, no. 1, pp. 77 - 86, 1989.

[13] S. Boyd, L. Xiao, A. Mutapcic, and J. Mattingley, "Notes on decomposition methods," Stanford University, Tech. Rep., 2008.

[14] S. J. Wright, "Partitioned dynamic programming for optimal control," SIAM Journal on Optimization, vol. 1, no. 4, pp. 620-642, 1991.

[15] D. Soudbakhsh and A. M. Annaswamy, "Parallelized model predictive control," in Proceedings of the American Control Conference, Washington, DC, USA, 2013, pp. 1715-1720.

[16] Y. Zhu and C. D. Laird, "A parallel algorithm for structured nonlinear programming," in Proceedings of the 5th International Conference on Foundations of Computer-Aided Process Operations, Boston, MA, USA, Jun. 2008, pp. 345-348.

[17] P. Reuterswärd, Towards Pseudospectral Control and Estimation. Licentiate's thesis, Lund University, 2012.

[18] S. Khoshfetrat Pakazad, "Divide and conquer: Distributed optimization and robustness analysis," Ph.D. dissertation, Linköping University, 2015.

[19] S. Khoshfetrat Pakazad, A. Hansson, M. S. Andersen, and I. Nielsen, "Distributed primal-dual interior-point methods for solving treestructured coupled convex problems using message-passing," Optimization Methods and Software, vol. 32, no. 3, pp. 401-435, 2017.

[20] I. Nielsen and D. Axehill, "An O(log N) parallel algorithm for Newton step computation in model predictive control," in Proceedings of the 19th IFAC World Congress, Cape Town, South Africa, Aug. 2014, pp. $10505-10511$.

[21] S. Boyd, N. Parikh, E. Chu, B. Peleato, and J. Eckstein, "Distributed optimization and statistical learning via the alternating direction method of multipliers," Foundations and Trends $®$ in Machine Learning, vol. 3, no. 1 , pp. $1-122,2011$

[22] I. Nielsen, "Structure-exploiting numerical algorithms for optimal control," Ph.D. dissertation, Linköping University, 2017.
[23] J. Nocedal and S. Wright, Numerical optimization. Springer-Verlag, 2006.

[24] D. Axehill, "Controlling the level of sparsity in MPC," Systems \& Control Letters, vol. 76, pp. 1-7, 2015.

[25] I. Nielsen and D. Axehill, "A parallel structure exploiting factorization algorithm with applications to model predictive control," in Proceedings of the 54th IEEE Conference on Decision and Control, Osaka, Japan, Dec. 2015, pp. 3932-3938.

[26] L. Lasdon, Optimization theory for large systems. Dover Publications, 1970.

[27] S. C. Chang, "A hierarchical temporal decomposition approach to long horizon optimal control problems," Ph.D. dissertation, The University of Connecticut, 1986

[28] J. M. Maciejowski, Predictive control with constraints. Prentice Hall, 2002.

[29] S. Boyd and L. Vandenberghe, Convex optimization. Cambridge University Press, 2004

[30] P. Lancaster and M. Tismenetsky, The Theory of Matrices: With Applications. Academic Press, 1985.

[31] W. J. Rugh, Linear System Theory, 2nd ed. Prentice-Hall, 1996

[32] P. Tøndel, T. A. Johansen, and A. Bemporad, "Further results on multiparametric quadratic programming," in Proceedings of the 42nd IEEE Conference on Decision and Control, Maui, HI, USA, Dec. 2003, pp. $3173-3178$

[33] J. L. Jerez, E. C. Kerrigan, and G. A. Constantinides, "A sparse and condensed QP formulation for predictive control of LTI systems," Automatica, vol. 48, no. 5, pp. 999 - 1002, 2012.

[34] D. P. Bertsekas, Dynamic Programming and Optimal Control, 2nd ed. Athena Scientific, 2000

[35] R. Penrose, "A generalized inverse for matrices," Mathematical Proceedings of the Cambridge Philosophical Society, vol. 51, no. 3, pp 406-413, 1955

[36] S. M. LaValle, Planning Algorithms. New York, NY, USA: Cambridge University Press, 2006.

[37] O. Ljungqvist, N. Evestedt, M. Cirillo, D. Axehill, and O. Holmer, "Lattice-based motion planning for a general 2-trailer system," arXiv preprint arXiv:1703.03616, 2017

[38] K. Bergman and D. Axehill, "Combining homotopy methods and numerical optimal control to solve motion planning problems," arXiv preprint arXiv:1703.07546, 2017

[39] K. J. Åström and B. Wittenmark, Computer controlled systems: theory and design. Prentice-Hall, 1984.

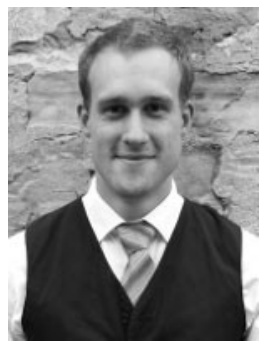

Isak Nielsen was born in Grimhult, Sweden, in 1987. He received his M.Sc. degree in Applied Physics and Electrical Engineering from Linköping University in Sweden in May 2012, where he was awarded the Tryggve-Holm medal for graduating with the highest GPA possible. In August 2012 he started his Ph.D. studies at Linköping University in Sweden, and in May 2017 he received his Ph.D. degree after successfully defended his thesis "Structure-Exploiting Numerical Algorithms for Optimal Control". His research interests are mainly within optimization for control and estimation, with focus on structureexploiting algorithms for model predictive control, moving horizon estimation and multiparametric programming.

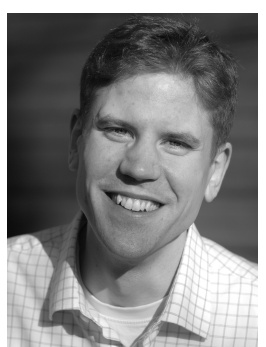

Daniel Axehill was born in Tranås, Sweden, in 1978. He received his M.Sc. degree in Applied Physics and Electrical Engineering in 2003. Furthermore, he received the degree of Lic.Eng. in Automatic Control in 2005 and the Ph.D. degree in Automatic Control in 2008. All three degrees are from Linköping University, Linköping, in Sweden. In year 2006 he spent three months at UCLA in Los Angeles. From January 2009 and until November 2010 he worked as a post-doc at the Automatic Control Laboratory at ETH Zurich. He is currently employed as an Associate Professor at the Division of Automatic Control at Linköping University. His research interests are related to optimization, optimal control, hybrid systems, signal processing, and applications of control. 